\title{
Representations of compact quantum groups and subfactors
}

\author{
Teodor Banica
}

Institut de Mathématiques de Luminy, case 930, F-13288 Marseille Cedex 9, France

E-mail: banica@iml.univ-mrs.fr

\begin{abstract}
We associate Popa systems (= standard invariants of subfactors, cf. [P3], $[\mathrm{P} 4]$ ) to the finite dimensional representations of compact quantum groups. We characterise the systems arising in this way: these are the ones which can be "represented" on finite dimensional Hilbert spaces. This is proved by an universal construction. We explicitely compute (in terms of some free products) the operation of going from representations of compact quantum groups to Popa systems and then back via the universal construction. This is related with our previous work [B2]. We prove a Kesten type result for the co-amenability of compact quantum groups, which allows us to compare it with the amenability of subfactors.
\end{abstract}

\section{Introduction}

After Jones' discovery of the index of subfactors and of the new invariant for links, the list of related theories and objects has considerably grown, one of them being the "quantum groups". The present work is an investigation of the relathionship between compact (and also discrete) quantum groups and subfactors. We construct and study three natural operations connecting such quantum groups and subfactors (see the abstract), and we discuss the amenability. In a certain sense, these are the only universal operations connecting representations of compact quantum groups and Popa systems (see the concluding remarks in the end of this section).

These considerations are mainly of theoretical nature and interest, but are also supposed to lead to explicit constructions of new quantum groups and subfactors. Let us mention that for the "classical" compact quantum groups (compact groups, duals of discrete groups, $q$-deformations) we obtain the subfactors in $[\mathrm{WS}],[\mathrm{P} 1]$, respectively those in $[\mathrm{X}]$ with $q>0$, togehter with some of their properties (e.g. the characterisation of the amenable 
ones). We also mention that when performing the above three operations with the simplest data, namely when the Popa system is the one generated by the Jones projections, or when the initial compact quantum group is just $\mathbf{S U}(2)$ (or more generally $\mathbf{S} \mathbf{U}_{q}(2)$ ), one obtains a free unitary quantum group, together with most of its main properties (Théorème 1 in [B2]).

A few words on the terminology. The paper is written using the formalism of Woronowicz algebras, which are the Hopf $\mathbf{C}^{*}$-algebras which correspond (by W2, for instance) to both notions of "algebras of continuous functions on compact quantum groups" and "C*-algebras of discrete quantum groups". Part of the results are better understood in terms of compact quantum groups, and the other part in terms of discrete quantum groups, so we have written this introductory section by using the more suggestive formalism of quantum groups associated with Woronowicz algebras.

It has to be mentioned that the locally compact quantum groups [BS] are known to be related to the depth 2 subfactors by a crossed product construction (see [EN] and the references therein). The point of view in this paper is completely different - in fact we relate the "theory of a single representation of compact quantum groups" with the "theory of the standard invariants of subfactors". By standard invariant we mean the lattice of higher relative commutants, which was shown to be a complete invariant for subfactors under amenability assumptions [P3]. When regarded as an abstract object satisfying the axioms in $[\mathbb{P} 4]$, it will be called Popa system.

We start from the general principle that if $\pi$ is a "representation of a quantum group" then the lattice $L(\pi)$ of algebras

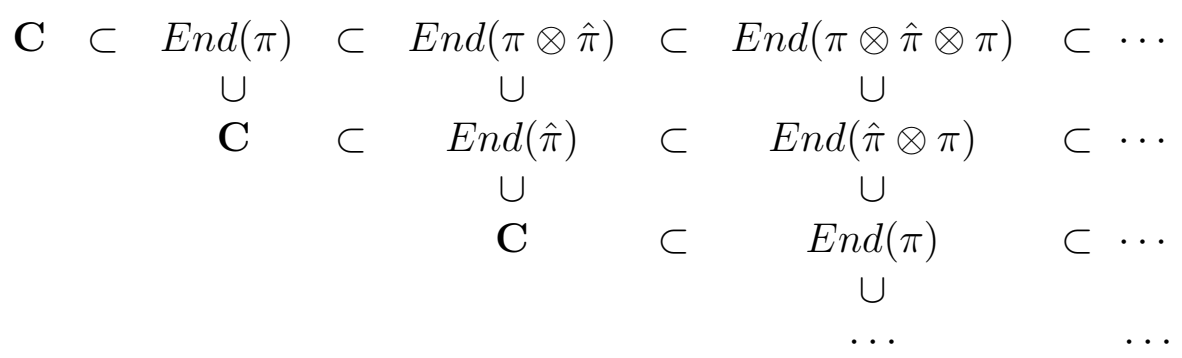

should be the standard invariant of a subfactor. An heuristic explanation for this fact is provided for instance by the Connes-Ocneanu bimodule picture of the standard invariant (see for instance [Bi]).

This general fact was intensively investigated for the quantum groups at roots of unity (which of course are not compact quantum groups), and 
a whole series of interesting subfactors was constructed in this way, see [We1], We2], [We3], [X] and also [J1], [TL].

The above general statement holds also when $\pi$ is a representation of a deformation $G_{q}$ of a compact group (with $q>0$ ) $\mathbb{X}$. Also the subfactors constructed by Wassermann in [W] and by Popa in [P1] have higher relative commutants of the form $L(\pi)$, with $\pi$ a representation of a compact group $G$ (resp. of a "dual" $\widehat{\Gamma}$ of a discrete group). These objects $G_{q}(q>0), G$ and $\widehat{\Gamma}$ are compact quantum groups in the sense of Woronowicz WW1, W2, W3 (for $G_{q}$, see [R2]), and in fact the following general result holds.

Theorem A. If $\pi$ is a finite dimensional unitary representation of a compact quantum group $G$, then the lattice $L(\pi)$ is a Popa system.

That is, $L(\pi)$ has to be the lattice of higher relative commutants of a subfactor (by $\mathbb{P} 4 \|$ ). The verification of Popa's axioms is very easy when the Haar measure $h \in C(G)^{*}$ is a trace; in the general case one has to perturbe everything by using the characters $f_{z} \in \mathcal{R}(G)^{*}$ describing the modular theory of the Haar measure W1]. The resulting index is the square of the quantum dimension of $\pi$.

There are several restrictions on the Popa systems arising in this way, and the most obvious one is that if $H$ is the Hilbert space where $\pi$ acts, then $\hat{\pi}$ acts on $\bar{H}, \pi \otimes \hat{\pi}$ acts on $H \otimes \bar{H}$ etc., so $L(\pi)$ has to be a sublattice of the following lattice $L(H)$ :

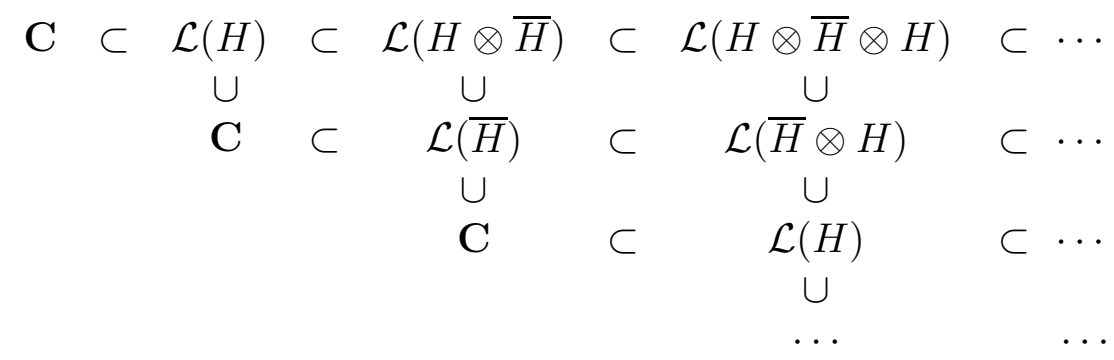

Theorem B. For any Popa system $\left(A_{i j}\right)_{0 \leq i \leq j<\infty}$ the following are equivalent: (i) there exists a compact quantum group $G$ and a finite dimensional representation $\pi$ of $G$ such that $\left(A_{i j}\right)_{0 \leq i \leq j<\infty}$ is equal to $L(\pi)$.

(ii) there exists a finite dimensional Hilbert space $H$ such that $\left(A_{i j}\right)_{0 \leq i \leq j<\infty}$ is a sublattice of $L(H)$, and such that the Jones projection $e_{2} \in A_{02}$ corresponds in this way to a rank one projection in $\mathcal{L}(H \otimes \bar{H})$. 
This shows for instance that the property of "coming from a representation of a compact quantum group" passes to sublattices.

(i) $\Longrightarrow$ (ii) is trivial, and the proof of the converse is based on the following observation. Associated to any compact quantum group $G$ is its monoidal category of representations $\operatorname{rep}(G)$, together with the forgetful functor $F_{G}: \operatorname{rep}(G) \rightarrow$ vect $_{\mathbf{C}}$. The Tannakian duality $\mathrm{W} 2$ says that the compact quantum groups are in one-to-one correspondence with the pairs (monoidal category, monoidal functor) satisfying a certain list of axioms. Now if $\pi$ is a representation of $G$, the algebras in $L(\pi)$ could be thought as a "piece of $\operatorname{rep}(G)$ "; and the embedding of $L(\pi)$ into $L(H)$ could be thought as the "corresponding piece of $F_{G}$ ". Thus what we have to do for proving (ii) $\Longrightarrow$ (i) is to construct a monoidal category and a monoidal functor when knowing "pieces" of them; and this kind of problem (see also [W2], [KW], [B1], [B2]) is well-known to be usually a combinatorial one.

The construction is not unique, and in fact we find the "universal" pair $(G, \pi)$ such that $\left(A_{i j}\right)_{0 \leq i \leq j<\infty}$ is equal to $L(\pi)$ (as sublattices of $L(H)$ ). The following question arises naturally: if $\left(A_{i j}\right)_{0 \leq i \leq j<\infty}$ itself is the Popa system associated to a pair $(G, \pi)$, who is universal pair that we construct ?

Theorem C. If $\pi$ is a representation of $G$ on $H$ and if $(\tilde{G}, \tilde{\pi})$ is the universal pair satisfying $L(\tilde{\pi})=L(\pi)$ as sublattices of $L(H)$ then there exists an (explicit) embedding $\widehat{\tilde{G}} \hookrightarrow \mathbf{Z} * \widehat{\pi(G)}$.

Here $*$ is the free product of discrete quantum groups Wn (see the section 5 for the rigorous statement, in terms of Hopf algebras). The proof uses an isomorphism criterion which relies on a result of free probability theory of Nica and Speicher [NS] and on the following idea from [B2]: the dimensions of the linear spaces $\operatorname{Hom}(r, p)$ with $r, p=$ tensor products between $\pi$ and $\hat{\pi}$ are exactly the $*$-moments of the character $\chi(\pi) \in C(G)$ with respect to the Haar measure $h \in C(G)^{*}$.

The above considerations give also a more conceptual proof for the result $A_{u}(F)_{\text {red }} \hookrightarrow \mathbf{C}^{*}(\mathbf{Z}) *_{\text {red }} A_{o}(F)$ from [B2]. In fact there are also other results from [B2] on $A_{u}(F)$ which extend to the algebras of the form $C(\tilde{G})$.

The last result is about amenability. For locally compact quantum groups the basic results on amenability were established by Blanchard [B], and in the discrete case a Kesten type result could be deduced from his work (this was explained to us by G. Skandalis). This result has several applications (see the section 6), one of them being: 
Theorem D. If $\pi$ is a representation of $G$ then $L(\pi)$ is amenable in the sense of PQ3 if and only if $K=(\pi \otimes \hat{\pi})(G)$ has the following properties: the dual $\widehat{K}$ is amenable, and the Haar measure $h \in C(K)^{*}$ is a trace.

This generalises some known results, and also shows that the index of an amenable lattice of the form $L(\pi)$ has to be the square of an integer.

Concluding remarks. The Theorems A,B,C could be interpreted in the following way. Fix $H$ and let $X$ be the set of pairs (compact quantum group, representation on $H$ ) and $Y$ be the set of pairs (Popa system, embedding into $L(H)$ ). Then A,B,C (and their proofs) give respectively: a surjection $X \rightarrow Y$; the universal section $Y \rightarrow X$; a description of the corresponding projection $X \rightarrow X$. It follows that the elements of $Y$ are in one-to-one correspondence with the elements in the image of the projection.

One remaining problem on the relationship between $X$ and Popa systems is to find the relationship between $Y$ and Popa systems.

One can show that the universal construction in the section 4 makes sense for any Popa system $\left(A_{i j}\right)_{0 \leq i \leq j<\infty}$, by giving abstract meanings to the operators $i$ and $p$ (e.g. small semicircles when working with planar diagrams [ए2]). If $\mathcal{R}_{\left(A_{i j}\right)_{0 \leq i \leq j<\infty}}$ is the monoidal category constructed in this way, then the above question of existence, uniqueness, classification of embeddings of $\left(A_{i j}\right)_{0 \leq i \leq j<\infty}$ into $L(H)$ 's is equivalent to the question of existence, uniqueness, classification of fiber functors on $\mathcal{R}_{\left(A_{i j}\right)_{0 \leq i \leq j<\infty}}$. While this can be done in certain cases, in general this is related to an unsolved problem, namely the generalisation of the theorems of Doplicher-Roberts [DR] and Deligne [D].

The paper is organised as follows. In the first section we recall Woronowicz' formalism, we discuss the notion of duality for unitary representations, and we give a list of relevant examples. In the second section we prove the Theorem A and we give some examples. In the third and the fourth section we prove the Theorem B. In the fifth and the sixth section we prove the Theorems C, respectively D.

Part of this work was done during my stay at UCLA and the University of Genève ; I would like to thank these institutions and Sorin Popa for their warm hospitality. I am also grateful to Dietmar Bisch and Georges Skandalis for useful discussions on subfactors and on quantum groups. 


\section{Duality for corepresentations}

The Woronowicz algebras are the Hopf $\mathbf{C}^{*}$-algebras which correspond (by [W1], [W2], [W3], [BS] etc.) to both notions of "algebras of continuous functions on compact quantum groups" and " $\mathbf{C}^{*}$-algebras of discrete quantum groups". They can be defined as being the bisimplifiable unital Hopf $\mathbf{C}^{*}$ algebras, see [W3]. One alternative definition, which will be used in this paper (see the Note below), is as follows.

Consider pairs $(A, u)$ consisting of a unital $\mathbf{C}^{*}$-algebra $A$ and a unitary matrix $u \in M_{n}(A)$ subject to the following conditions (ए1], def. 1.1) :

(w1) the coefficients of $u$ generate in $A$ a dense $*$-subalgebra, called $A_{s}$.

(w2) there exists a $\mathbf{C}^{*}$-morphism $\delta: A \rightarrow A \otimes_{\min } A$ sending $u_{i j} \mapsto$ $\sum u_{i k} \otimes u_{k j}$ for any $1 \leq i, j \leq n$.

(w3) there exists a linear antimultiplicative map $\kappa: A_{s} \rightarrow A_{s}$ such that $\kappa\left(\kappa\left(a^{*}\right)^{*}\right)=a$ for all $a \in A_{s}$ and such that $(i d \otimes \kappa) u=u^{-1}$.

If $(A, u)$ and $(B, v)$ are as above, one can define the morphisms from $(A, u)$ to $(B, v)$ to be the $\mathbf{C}^{*}$-morphisms $f: A \rightarrow B$ satisfying $\delta_{B} f=(f \otimes$ f) $\delta_{A}$. The Woronowicz algebras are by definition the inductive limits in the corresponding category of pairs $(A, u)$ satisfying (w1-3).

If $(A, u)$ satisfies $(w 1-3)$ then the dense subalgebra $A_{s}$ is an involutive Hopf $\mathbf{C}$-algebra with comultiplication $\delta$, antipode $\kappa$ and counit defined by $\varepsilon: u_{i j} \mapsto \delta_{i j}$. Recall that the finite dimensional corepresentations of $A_{s}$ are the elements $r \in \mathcal{L}(V) \otimes A_{s}$ satisfying $(i d \otimes \delta) r=r_{12} r_{13}$, where $V$ is a finite dimensional complex vector space (for instance the matrix $u$ is a unitary corepresentation of $A_{s}$ on $\mathbf{C}^{n}$, called the fundamental corepresentation of $(A, u))$. It was proved in W1] that every non degenerate such corepresentation is equivalent to a unitary corepresentation, and is completely reducible. The Hopf algebra $A_{s}$ has then a (cosemisimple) decomposition

$$
A_{s}=\bigoplus_{r \in \operatorname{Irr}(A)} A(r)
$$

where $\operatorname{Irr}(A)$ is the set of equivalence classes of finite dimensional irreducible corepresentations of $A_{s}$, and for every (class of) corepresentation $r \in \mathcal{L}(V) \otimes$ $A_{s}$ its linear space of coefficients is $A(r):=\left\{(\varphi \otimes i d) r \mid \varphi \in \mathcal{L}(V)^{*}\right\}$.

These results extend to all the Woronowicz algebras (see [W3], [BS], [Wn]).

It is easy to see that the finite dimensional corepresentations of $C(G)$ correspond to the finite dimensional representations of $G$ (for $G=$ compact 
group) and that the finite dimensional irreducible corepresentations of $\mathbf{C}^{*}(\Gamma)$ correspond to the elements of $\Gamma$ (for $\Gamma=$ discrete group).

Note. If $u$ is a finite dimensional unitary corepresentation of a Woronowicz algebra $B$, and if $A$ is the $\mathbf{C}^{*}$-subalgebra of $B$ generated by the coefficients of $u$ then $(A, u)$ satisfies the conditions (w1-3). As we will be interested in the theory of a single corepresentation of a Woronowicz algebra, all the Woronowicz algebras $A$ we consider in this paper may be supposed to satisfy the conditions (w1-3) for some matrix $u \in M_{n}(A)$, i.e. may be supposed to be as in [W1. Note that these Woronowicz algebras are exactly the ones whose associated compact quantum groups are "compact matrix quantum groups", or, equivalently, whose associated discrete quantum groups are "of finite type".

One of the main results of W1 was the construction of a canonical family of characters $\left\{f_{z}\right\}_{z \in \mathbf{C}}$ of $A_{s}$, which describe the modular theory of the Haar measure $h \in A^{*}$. The $f_{z}$ 's have the following properties (where $*$ denotes the convolutions over the Hopf algebra $A_{s}$ ):

(f1) $f_{z} * f_{z^{\prime}}=f_{z+z^{\prime}}, \forall z, z^{\prime} \in \mathbf{C}$ and $f_{0}=\varepsilon$ (the counit of $A_{s}$ ).

(f2) $f_{z} \kappa(a)=f_{-z}(a), f_{z}\left(a^{*}\right)=\overline{f_{-\bar{z}}(a)}, \forall a \in A_{s}$ and $z \in \mathbf{C}$.

(f3) $\kappa^{2}(a)=f_{-1} * a * f_{1}, \forall a \in A_{s}$.

(f4) $h(a b)=h\left(b f_{1} * a * f_{1}\right), \forall a, b \in A_{s}$.

If $v \in \mathcal{L}(H) \otimes A_{s}$ is a finite dimensional unitary corepresentation of $A_{s}$ then the restriction of $f_{z}$ to the space of coefficients of $v$ can be computed by using the formula $\left(i d \otimes f_{z}\right) v=Q_{v}^{2 z}$, where

$$
Q_{v}:=\left(i d \otimes f_{\frac{1}{2}}\right) v \in \mathcal{L}(H)
$$

(this follows from $(f 1))$. In the Lemma 1.1 we list the properties of the operator $Q_{v}$, and in the Lemma 1.2 we give a characterisation of $Q_{v}$.

Notations. If $H$ is a finite dimensional Hilbert space, $\bar{H}$ is its complex conjugate. We will use the identifications $\overline{\bar{H}}=H$ and $\overline{H \otimes K}=\bar{K} \otimes \bar{H}$ (but not $H \simeq \bar{H}$, even if $\left.H=\mathrm{C}^{n}\right)$. The transposition $\mathcal{L}(H) \rightarrow \mathcal{L}(\bar{H})$ will be denoted by $a \mapsto t(a)$ or $a \mapsto a^{t}$.

Lemma 1.1 If $v \in \mathcal{L}(H) \otimes A_{s}$ is a finite dimensional unitary corepresentation of $A_{s}$ then $Q_{v}$ has the following properties :

(i) $Q_{v}>0$. 
(ii) $Q_{v} \in \operatorname{End}(v)^{\prime}$.

(iii) $\operatorname{Tr}\left(Q_{v}^{2}\right.$. $)=\operatorname{Tr}\left(Q_{v}^{-2}\right.$.) on $\operatorname{End}(v)$.

(iv) $Q_{v}^{2} \in \operatorname{Hom}\left(v,\left(i d \otimes \kappa^{2}\right) v\right)$.

(v) $Q_{v}^{t} \bar{v}\left(Q_{v}^{t}\right)^{-1}$ is unitary.

Proof. We prove firstly (v). We have

$$
Q_{v}^{t} \bar{v}\left(Q_{v}^{t}\right)^{-1}=\left[\left(t \otimes f_{\frac{1}{2}}\right) v\right][(t \otimes \kappa) v]\left[\left(t \otimes f_{-\frac{1}{2}}\right) v\right]=(t \otimes j) v
$$

where $j: A_{s} \rightarrow A_{s}$ is the linear map $x \mapsto f_{\frac{1}{2}} * \kappa(x) * f_{-\frac{1}{2}}$. It's easy to see using $(f 1, f 2, f 3)$ that $j$ is antimultiplicative, and that it commutes with the involution of $A_{s}$. Thus $t \otimes j: \mathcal{L}(H) \otimes A_{s} \rightarrow \mathcal{L}(\bar{H}) \otimes A_{s}$ is an antihomorphism of $*$-algebras, so it maps unitaries to unitaries and this proves $(\mathrm{v})$.

If $v$ is irreducible then (ii) is trivial and (i,iii,iv) follow from $(f 1-f 4)$, see the section 5 in W1. The results (i-iv) can be extended to the general case by using cosemisimplicity : by [W] we may suppose that $v=\sum m_{i} v_{i}$ with $m_{i} \in \mathbf{N}$ and $v_{i} \in \mathcal{L}\left(H_{i}\right) \otimes A_{s}$ irreducible and disjoint. As $m_{i} v_{i}=v_{i} \otimes\left(m_{i} 1\right)$ acts on $\mathcal{L}\left(H_{i}\right) \otimes \mathcal{L}\left(\mathbf{C}^{m_{i}}\right)$ for every $i$ we may suppose that $H=\oplus_{i}\left(H_{i} \otimes \mathbf{C}^{m_{i}}\right)$. With respect to this decomposition we have

$$
\operatorname{End}(v)=\bigoplus_{i} i d_{H_{i}} \otimes \mathcal{L}\left(\mathbf{C}^{m_{i}}\right)
$$

and $Q_{v}=\sum_{i} Q_{v_{i}} \otimes i d_{\mathbf{C}^{m_{i}}}$, and this proves (i,ii,iv). Also if $x \in \operatorname{End}(v)$ then $x$ is of the form $\sum_{i} i d_{H_{i}} \otimes x_{i}$, so

$$
\operatorname{Tr}\left(x Q_{v}^{2}\right)=\sum_{i} \operatorname{Tr}\left(Q_{v_{i}}^{2}\right) \operatorname{Tr}\left(x_{i}\right)=\sum_{i} \operatorname{Tr}\left(Q_{v_{i}}^{-2}\right) \operatorname{Tr}\left(x_{i}\right)=\operatorname{Tr}\left(x Q_{v}^{-2}\right)
$$

and this proves (iii).

Lemma 1.2 If $v \in \mathcal{L}(H) \otimes A_{s}$ is a finite dimensional unitary corepresentation of $A_{s}$ and $Q \in \mathcal{L}(H)$ is a positive operator such that $\operatorname{Tr}\left(Q^{2}.\right)=\operatorname{Tr}\left(Q^{-2}\right.$.) on $\operatorname{End}(v)$, then the following are equivalent:

(i) $Q=Q_{v}$.

(ii) $Q^{t} \bar{v}\left(Q^{t}\right)^{-1}$ is unitary.

(iii) $Q^{2} \in \operatorname{Hom}\left(v,\left(i d \otimes \kappa^{2}\right) v\right)$.

(iv) $Q Q_{v}^{-1} \in \operatorname{End}(v)$. 
Proof. $(i \Longrightarrow$ ii) was part of the preceding lemma.

(ii $\Longrightarrow$ iii) Using the formula $(i d \otimes \kappa) r=r^{*}$ for the unitary corepresentations $r:=v$ and $r:=Q^{t} \bar{v}\left(Q^{t}\right)^{-1}$ we get $(i d \otimes \kappa) v=v^{*}$ and $(i d \otimes \kappa) \bar{v}=$ $\left(Q^{t}\right)^{-2} v^{t}\left(Q^{t}\right)^{2}$, so $\left(i d \otimes \kappa^{2}\right) v=Q^{2} v Q^{-2}$.

(iii $\Longrightarrow i v)$ We have $Q^{2}, Q_{v}^{2} \in \operatorname{Hom}\left(v,\left(i d \otimes \kappa^{2}\right) v\right)$, so $Q^{2}=Q_{v}^{2} x$ for some $x \in \operatorname{End}(v)$. As $Q_{v}$ commutes with $x$, the result follows by taking square roots.

$(i v \Longrightarrow i)$ By using a decomposition $v=\sum v_{i} \otimes\left(m_{i} 1\right)$ with $v_{i} \in \mathcal{L}\left(H_{i}\right) \otimes A_{s}$ as in the proof of the preceding lemma, we may write $Q Q_{v}^{-1}=\sum_{i} i d_{H_{i}} \otimes x_{i}$ with $x_{i} \in \mathcal{L}\left(\mathbf{C}^{m_{i}}\right)$. Thus $Q=\sum Q_{v_{i}} \otimes x_{i}$, so for every $i$ the restriction to $\operatorname{End}\left(v_{i} \otimes\left(m_{i} 1\right)\right)=\mathcal{L}\left(\mathbf{C}^{m_{i}}\right)$ of the linear form $\operatorname{Tr}\left(Q^{2}\right.$.) (resp. $\left.\operatorname{Tr}\left(Q^{-2}.\right)\right)$ is $\operatorname{Tr}\left(Q_{v_{i}}^{2}\right) \operatorname{Tr}\left(x_{i}^{2}\right.$.) (resp. $\left.\operatorname{Tr}\left(Q_{v_{i}}^{-2}\right) \operatorname{Tr}\left(x_{i}^{-2}.\right)\right)$. As $\operatorname{Tr}\left(Q_{v_{i}}^{2}\right)=\operatorname{Tr}\left(Q_{v_{i}}^{-2}\right)$, we have $\operatorname{Tr}\left(x_{i}^{2}.\right)=\operatorname{Tr}\left(x_{i}^{-2}\right.$.) on $\mathcal{L}\left(\mathbf{C}^{m_{i}}\right)$, so $x_{i}^{2}=x_{i}^{-2}$ for every $i$. But all $x_{i}$ 's are positive, so they have to be the identities, and $Q=Q_{v}$.

Definition 1.1 (cf. Lemma 1.1) If $v \in \mathcal{L}(H) \otimes A_{s}$ is a finite dimensional unitary corepresentation of $A_{s}$, the positive number

$$
d(v):=\operatorname{Tr}\left(Q_{v}^{2}\right)=\operatorname{Tr}\left(Q_{v}^{-2}\right)=\left(\operatorname{Tr} \otimes f_{1}\right) v=f_{1} \chi(v)
$$

is called the quantum dimension of $v$. The linear form

$$
\tau_{v}: x \mapsto d(v)^{-1} \operatorname{Tr}\left(Q_{v}^{2} x\right)=d(v)^{-1} \operatorname{Tr}\left(Q_{v}^{-2} x\right)
$$

is called the canonical trace on End $(v)$. The unitary corepresentation

$$
\hat{v}:=\left(Q_{v}\right)^{t} \bar{v}\left(Q_{v}^{t}\right)^{-1}=\left(t \otimes f_{\frac{1}{2}} * \kappa(.) * f_{-\frac{1}{2}}\right) v
$$

is called the canonical dual of $v$.

Note that $\tau_{v}$ is a faithful positive unital trace on the $\mathbf{C}^{*}$-algebra $\operatorname{End}(v)$. By the Cauchy-Schwartz inequality applied to the eigenvalues of $Q_{v}^{2}$ and of $Q_{v}^{-2}$ we see that $d(v) \geq \operatorname{dim}(H)$, with equality iff $Q_{v}=i d_{H}$.

We will give now a list of examples of Woronowicz algebras, with computation of the objects in the above definition, and/or of the fusion semiring. We recall that if $A$ is a Woronowicz algebra, the fusion semiring $R^{+}(A)$ is the semiring whose elements are equivalence classes of finite dimensional corepresentations of $A$, and whose operations are the sum and tensor product of 
(classes of) corepresentations. Its computability is of course the most important requirement for having the computability of the (fusion algebra of) Popa systems associated to $A$.

The examples below illustrate most of the phenomena arising when comparing Woronowicz algebras with Popa systems, and will be quoted along the rest of the paper.

Example 1.1. If $A$ is a Woronowicz algebra then the following conditions are equivalent (cf. the formulas $(f 1-4)$ see $[\mathrm{W} 1],[\mathrm{BS} \|)$ :

- the Haar measure $h \in A^{*}$ is a trace.

- the square of the antipode $\kappa$ is the identity of $A_{s}$.

- all the $f_{z}$ 's are equal to the counit.

This happens for instance if $A$ is of the form $C(G)$ with $G$ compact group, or $\mathbf{C}^{*}(\Gamma)$ with $\Gamma$ discrete group, or when $A$ is finite dimensional. In this case it is clear that $Q_{v}=i d_{H}, d(v)=\operatorname{dim}(H)$, and $\hat{v}=\bar{v}$ for any unitary corepresentation $v \in \mathcal{L}(H) \otimes A_{s}$.

Example 1.2. The $q$-deformation of the $\mathbf{C}^{*}$-algebras $C(G)$, with $G$ compact classical Lie group and $q>0$ was started by Woronowicz, and completed by Rosso [R2, via a beautiful application of the Tannakian duality W2. By [R1], [R2] the fusion semiring is invariant under $q$-deformations.

If $\mu \in[-1,1]-\{0\}$ and $u$ is the fundamental representation of $\mathbf{S}_{\mu} \mathbf{U}(2)$ then $Q_{u}=\operatorname{diag}\left(|\mu|^{-1},|\mu|\right)$ (cf. the Appendix of W1]).

Example 1.3. For $n \in \mathbf{N}$ and $F \in \mathbf{G L}(n, \mathbf{C})$ satisfying $F \bar{F} \in \mathbf{R} I_{n}$ let $A_{o}(F)$ be the universal $\mathbf{C}^{*}$-algebra generated by the entries of a $n \times n$ matrix $u$ with the relations $u=F \bar{u} F^{-1}=$ unitary. This Woronowicz algebra was introduced in VDW as to represent the "free analogue of $\mathbf{O}(n)$ " and its fusion semiring was shown in [B1] to be isomorphic to the one of $\mathbf{S U}(2)$. Moreover, by combining this result with [KW] one finds that the category of corepresentations of $A_{o}(F)$ is always monoidal equivalent to the category of corepresentations of $\mathbf{S}_{\mu} \mathbf{U}(2)$ for some $\mu \in[-1,1]-\{0\}$. This is a relevant example for the considerations in the end of the Introduction.

As a matter of fact, we mention that the Woronowicz algebras $A_{o}(F)$ are exactly the "compact forms" of the Gurevich's quantizations of $\mathbf{S L}(2, \mathbf{C})$ G].

Example 1.4. For $n \in \mathbf{N}$ and $F \in \mathbf{G L}(n, \mathbf{C})$ let $A_{u}(F)$ be the universal $\mathrm{C}^{*}$-algebra generated by the entries of a $n \times n$ matrix $u$ with the relations $u$ unitary, $F \bar{u} F^{-1}$ unitary ; these Woronowicz algebras correspond to both notions of "algebras of continuous functions on the free unitary groups" and 
"C $\mathrm{C}^{*}$-algebras of the free free groups" (sic) [NDW]. The fusion semiring of $A_{u}(F)$ was computed in [B2]. Using (ii) $\Rightarrow(i)$ in the Lemma 1.2 it's easy to see that $Q_{u}$ is a scalar multiple of the transpose of $\sqrt{F^{*} F}$.

Thus if $n \in \mathbf{N}$ and $Q \in M_{n}(\mathbf{C})$ is a positive diagonal matrix such that $\operatorname{Tr}\left(Q^{2}\right)=\operatorname{Tr}\left(Q^{-2}\right)$, then for $\left(A_{u}(Q), u\right)$ we have $Q_{u}=Q$ (note : one can show that given any $n \in \mathbf{N}$ and $F \in \mathbf{G L}(n, \mathbf{C})$ there exists such a matrix $Q$ such that $A_{u}(F)$ is isomorphic to $A_{u}(Q)$, and that $Q$ is unique up to $Q \mapsto Q^{-1}$ and up to the permutation of its diagonal entries ; this classification of the algebras $A_{u}(F)$ could be easily deduced from the results in [B2] and from the above computation of $Q_{u}$ ).

Lemma 1.3 (i) $Q_{\hat{v}}=\left(Q_{v}^{t}\right)^{-1}, \hat{\hat{v}}=v$ and $d(\hat{v})=d(v)$.

(ii) $Q_{v \otimes w}=Q_{v} \otimes Q_{w}, v \widehat{\otimes} w=\hat{w} \otimes \hat{v}$ and $d(v \otimes w)=d(v) d(w)$.

(iii) $Q_{v+w}=\operatorname{diag}\left(Q_{v}, Q_{w}\right), v \widehat{+w}=\hat{v}+\hat{w}$ and $d(v+w)=d(v)+d(w)$.

Proof. The formulas for the quantum dimensions and for the canonical duals follow easily from the formulas for the $Q$ 's, which we will now prove. Firstly, $Q_{v+w}=\operatorname{diag}\left(Q_{v}, Q_{w}\right)$ is clear and $Q_{v \otimes w}=Q_{v} \otimes Q_{w}$ follows from the fact that $f_{\frac{1}{2}}$ is a character. Also using (f1) and (f2) we get that

$$
\begin{aligned}
& Q_{\hat{v}}=\left(i d \otimes f_{\frac{1}{2}}\right)\left(t \otimes f_{\frac{1}{2}} * \kappa(.) * f_{-\frac{1}{2}}\right) v=\left(t \otimes f_{\frac{1}{2}}\left(f_{\frac{1}{2}} * \kappa(.) * f_{-\frac{1}{2}}\right)\right) v= \\
& \left(t \otimes\left(f_{\frac{1}{2}} * f_{\frac{1}{2}} * f_{-\frac{1}{2}}\right) \kappa\right) v=\left(t \otimes f_{\frac{1}{2}} \kappa\right) v=\left(t \otimes f_{-\frac{1}{2}}\right) v=\left(Q_{v}^{t}\right)^{-1} \square
\end{aligned}
$$

If $v$ is a finite dimensional unitary corepresentation of $A_{s}$ then the contragradient $v^{c}$, the complex conjugate $\bar{v}$ and the canonical dual $\hat{v}$ are of course in the same equivalence class, which is dual to the equivalence class of $v$. In the next proposition we state the precise (spatial) form of duality between $v$ and $\hat{v}$ (see the remark below).

Notation. If $H$ is a finite dimensional Hilbert space $\xi: \mathcal{L}(H) \simeq H \otimes \bar{H}$ is the canonical linear map.

Proposition 1.1 For any finite dimensional unitary corepresentation $v \in$ $\mathcal{L}(H) \otimes A_{s}$ define $i_{v} \in \mathcal{L}(\mathbf{C}, H \otimes \bar{H})$ by $1 \mapsto \xi\left(Q_{v}\right)$. Define also $p_{v}:=i_{\hat{v}}^{*}$. Then the following "duality formulas" hold:

$(d 1) i_{v} \in \operatorname{Hom}(1, v \otimes \hat{v})$ and $p_{v} \in \operatorname{Hom}(\hat{v} \otimes v, 1)$.

(d2) $\left(i d_{v} \otimes p_{v}\right)\left(i_{v} \otimes i d_{v}\right)=i d_{v}$ and $\left(p_{v} \otimes i d_{\hat{v}}\right)\left(i d_{\hat{v}} \otimes i_{v}\right)=i d_{\hat{v}}$.

(d3) $p_{\hat{v}}=i_{v}^{*}, p_{v}=i_{\hat{v}}^{*}$ and $p_{v} i_{\hat{v}}=p_{\hat{v}} i_{v}=d(v)=d(\hat{v})$.

(di) $p_{r \otimes s}=p_{s}\left(i d_{\hat{s}} \otimes p_{r} \otimes i d_{s}\right)$ and $i_{r \otimes s}=\left(i d_{r} \otimes i_{s} \otimes i d_{\hat{r}}\right) i_{r}, \forall r, s$. 
Proof. (d3) is trivial, and (d/4) follows from the Lemma 1.3 (ii). Let us choose an orthogonal basis $\left\{e_{1}, \ldots, e_{n}\right\}$ of $H$ consisting of eigenvectors of $Q_{v}$, so that $Q_{v}=\sum Q_{i i} e_{i i}$. Then $<\left(i d_{v} \otimes p_{v}\right)\left(i_{v} \otimes i d_{v}\right) x, y>$ is equal to

$<\xi\left(Q_{v}\right) \otimes x, y \otimes \xi\left(Q_{\hat{v}}\right)>=\sum<Q_{i i} e_{i} \otimes \bar{e}_{i} \otimes x, y \otimes Q_{j j}^{-1} \otimes \bar{e}_{j} \otimes e_{j}>=<x, y>$

for any $x, y \in H$, and (d2) follows. It remains to verify that $i_{v} \in \operatorname{Hom}(1, v \otimes$ $\hat{v})$ for any $v$; the other assertion $p_{v} \in \operatorname{Hom}(\hat{v} \otimes v, 1)$ will follow from $p_{v}=i_{\hat{v}}^{*}$ and from $i_{\hat{v}} \in \operatorname{Hom}(1, \hat{v} \otimes v)$. We have $i_{v}(1)=\sum Q_{i i} e_{i} \otimes \bar{e}_{i}$, and if $\zeta:=\sum e_{i} \otimes \bar{e}_{i}$ then

$$
(v \otimes \bar{v})(\zeta \otimes 1)=\sum_{i, k, a} e_{i} \otimes \bar{e}_{k} \otimes v_{i a} v_{k a}^{*}=\sum_{i, k} e_{i} \otimes \bar{e}_{k} \otimes \delta_{i k} 1=(\zeta \otimes 1)
$$

As $\bar{v}=\left(Q_{v}^{-1}\right)^{t} \hat{v}\left(Q_{v}\right)^{t}$ we get $(v \otimes \hat{v})\left(\sum Q_{i i} e_{i} \otimes \bar{e}_{i} \otimes 1\right)=\left(\sum Q_{i i} e_{i} \otimes \bar{e}_{i} \otimes 1\right)$, i.e. $i_{v} \in \operatorname{Hom}(1, v \otimes \hat{v})$.

Remark. Recall that in a monoidal category a duality $\left(X, Y, i_{X}, p_{X}\right)$ consists of two objects $X, Y$ and two morphisms $i_{X} \in \operatorname{Hom}(1, X \otimes Y)$, $p_{X} \in \operatorname{Hom}(Y \otimes X, 1)$ such that the compositions

$$
\begin{aligned}
& X=1 \otimes X \stackrel{i_{X} \otimes i d}{\longrightarrow} X \otimes Y \otimes X \stackrel{i d \otimes p_{X}}{\longrightarrow} X \otimes 1=X \\
& Y=Y \otimes 1 \stackrel{i d \otimes i_{X}}{\longrightarrow} Y \otimes X \otimes Y \stackrel{p_{X} \otimes i d}{\longrightarrow} 1 \otimes Y=Y
\end{aligned}
$$

are equal to $i d_{X}$ and $i d_{Y}$ correspondingly (in this case Frobenius reciprocity holds, etc. - see for instance [KW]). Thus $(d 1, d 2)$ show that $\left(v, \hat{v}, i_{v}, p_{v}\right)$ and $\left(\hat{v}, v, i_{\hat{v}}, p_{\hat{v}}\right)$ are dualities, i.e. that $\hat{v}$ is both a right and a left dual for $v$ is the monoidal category of finite dimensional corepresentations of $A$.

\section{Algebras of symmetries}

We use the following notation for tensor products and related lattices.

Notations. Let $\mathbf{N}^{* 2}=<\alpha, \beta>$ be the free monoid consisting of words in $\alpha, \beta$, plus the empty word denoted by $e$. We define an involution - on $\mathbf{N}^{* 2}$ by $\hat{\alpha}=\beta, \hat{\beta}=\alpha$ and antimultiplicativity.

If $\left(\mathcal{C}, \otimes, 1,{ }^{\wedge}\right)$ is an involutive monoid, and if $A \in \mathcal{C}, x \in \mathbf{N}^{* 2}$ we denote by $A^{\otimes x}$ the image of $x$ by the unique morphism $\mathbf{N}^{* 2} \rightarrow \mathcal{C}$ sending $\alpha \mapsto A$. 
If $0 \leq i \leq j$ are integers we define $[i, j] \in \mathbf{N}^{* 2}$ to be $(\alpha \beta)^{\frac{j-i}{2}}$ if $i, j$ are even, $(\beta \alpha)^{\frac{j-i}{2}}$ if $i, j$ are odd, $(\alpha \beta)^{\frac{j-i-1}{2}} \alpha$ if $i$ is even and $j$ is odd, and $\beta(\alpha \beta)^{\frac{j-i-1}{2}}$ if $i$ is odd and $j$ is even.

We will use this notation when $(\mathcal{C}, \otimes, 1)$ is the monoidal category of unitary corepresentations of a Woronowicz algebra, with ${ }^{\wedge}=$ the canonical dual, or when $(\mathcal{C}, \otimes, 1)$ is the monoidal category of complex vector spaces, with ${ }^{\wedge}=$ complex conjugation. Thus the lattices $L(\pi)$ and $L(H)$ in the Introduction are equal to $\operatorname{End}\left(\pi^{\otimes[i, j]}\right)_{0 \leq i \leq j<\infty}$ and $\operatorname{End}\left(H^{\otimes[i, j]}\right)_{0 \leq i \leq j<\infty}$ respectively.

Recall that a standard $\lambda$-lattice of commuting squares (or Popa system) is a system $\left(A_{i j}\right)_{0 \leq i \leq j<\infty}$ of finite dimensional $\mathbf{C}^{*}$-algebras with $A_{i i}=\mathbf{C}$, $A_{i j} \subset A_{k l}$ for $k \leq i, j \leq l$, and with a given faithful trace $\tau$ on $\cup_{i, j} A_{i j}$ satisfying the following properties (see the section 1 of [P4]) :

The commuting square condition. $E_{A_{i j}} E_{A_{k l}}=E_{A_{k l}} E_{A_{i j}}=E_{A_{r s}}$ where $r=$ $\max \{i, k\}, s=\min \{j, l\}$ and $E_{B}$ is the $\tau$-preserving conditional expectation onto $B$.

Jones conditions. There exists a representation of the $\lambda$-sequence of Jones projections $\left\{e_{i}\right\}_{i \geq 2}$ in $\cup_{i, j} A_{i j}$ such that $e_{j} \in A_{i-2, k}, \forall 2 \leq i \leq j \leq k$ and

$e_{j+1} x e_{j+1}=E_{A_{i, j-1}}(x) e_{j+1}, \forall i \leq j-1$ and $x \in A_{i j}$.

$e_{i+1} x e_{i+1}=E_{A_{i+1, j}}(x) e_{i+1}, \forall i+1 \leq j$ and $x \in A_{i j}$.

Markov conditions.

$\lambda^{-1} E_{A_{i, j+1}}\left(x e_{j+2}\right) e_{j+2}=x e_{j+2}, \forall j \geq i$ and $x \in A_{i, j+2}$.

$\lambda^{-1} E_{A_{i+1, j}}\left(x e_{i+2}\right) e_{i+2}=x e_{i+2}, \forall j \geq i+2$ and $x \in A_{i, j}$.

Commutation relations. $\left[A_{i j}, A_{k l}\right]=0, \forall i \leq j \leq k \leq l$.

Theorem 2.1 If $v$ is a finite dimensional unitary corepresentation of a Woronowicz algebra then the lattice $\operatorname{End}\left(v^{\otimes[i, j]}\right)_{0 \leq i \leq j<\infty}$ (together with the canonical traces from the def. 1.1) is a Popa $\lambda$-system, with $\lambda=d(v)^{-2}$.

Proof. We have to verify the above four axioms. The Commutation relations are clear. Note that all the inclusions between the algebras in the lattice are of the form

$$
\operatorname{End}(a) \hookrightarrow \operatorname{End}(r \otimes a \otimes w), x \mapsto i d_{r} \otimes x \otimes i d_{w}
$$

Let us define a linear map $E_{r, a, w}: \operatorname{End}(r \otimes a \otimes w) \rightarrow \operatorname{End}(a)$ by

$$
x \mapsto d(r)^{-1} d(w)^{-1}\left(p_{r} \otimes i d_{a} \otimes p_{\hat{w}}\right)\left(i d_{\hat{r}} \otimes x \otimes i d_{\hat{w}}\right)\left(i_{\hat{r}} \otimes i d_{a} \otimes i_{w}\right)
$$


By using (d4) we get

$$
E_{r, a, w} E_{r^{\prime}, r \otimes a \otimes w, w^{\prime}}=E_{r^{\prime} \otimes r, a, w \otimes w^{\prime}} \quad \forall r^{\prime}, r, a, w, w^{\prime}
$$

We verify now that $E_{r, a, w}$ is the conditional expectation, i.e. that

$$
\begin{gathered}
E_{r, a, w}\left(i d_{r} \otimes x \otimes i d_{w}\right)=x \\
E_{r, a, w}\left[\left(i d_{r} \otimes x \otimes i d_{w}\right) y\left(i d_{r} \otimes z \otimes i d_{w}\right)\right]=x E_{r, a, w}(y) z \\
\tau_{r \otimes a \otimes w}=\tau_{a} E_{r, a, w}
\end{gathered}
$$

$\forall x, z \in \operatorname{End}(a)$ and $y \in \operatorname{End}(r \otimes a \otimes w)$. Indeed, the first formula follows from (d3), the second one is clear by definition of $E_{r, a, w}$, and the third one follows from $\tau_{v}=E_{1,1, v}=E_{v, 1,1}$ for any $v$ and from $(\star)$. The commuting square axiom follows now from $(\star)$. Define now for every $k \geq 1$

$$
e_{2 k}:=d(v)^{-1} i_{v} p_{\hat{v}} \in \operatorname{End}(v \otimes \hat{v}), \quad e_{2 k+1}:=d(v)^{-1} i_{\hat{v}} p_{v} \in \operatorname{End}(\hat{v} \otimes v) .
$$

By (d3), the $e_{k}$ 's are selfadjoint projections. Using (d2) it's easy to see that $\left\{e_{2}, e_{3}, \ldots\right\}$ is a representation of the $\lambda$-sequence of Jones projections, with $\lambda:=d(v)^{-2}$. We will prove the other two axioms for the horizontal inclusions (same proof for the vertical ones). Note that every pair of consecutive horizontal inclusions is of the form

$$
\operatorname{End}(a) \hookrightarrow \operatorname{End}(a \otimes w) \hookrightarrow \operatorname{End}(a \otimes w \otimes \hat{w})
$$

with $w=v$ or $\hat{v}$, and the Jones projection in $\operatorname{End}(a \otimes w \otimes \hat{w})$ is $e:=$ $d(w)^{-1} i d_{a} \otimes i_{w} p_{\hat{w}}$. The Jones condition, namely

$$
e\left(x \otimes i d_{\hat{w}}\right) e=\left(E_{1, a, w}(x) \otimes i d_{w \otimes \hat{w}}\right) e, \forall x \in \operatorname{End}(a \otimes w)
$$

is trivial from the definitions of $e$ and $E_{1, a, w}$. The Markov condition follows by applying $(d 2, d 3)$ several times - if $x \in \operatorname{End}(a \otimes w \otimes \hat{w})$ then

$$
\begin{aligned}
& d(w)^{2}\left(E_{1, a \otimes w, \hat{w}}(x e) \otimes i d_{\hat{w}}\right) e= \\
& {\left[\left(i d_{a \otimes w} \otimes p_{w}\right)\left(x \otimes i d_{w}\right)\left(i d_{a} \otimes i_{w} \otimes i d_{w}\right) \otimes i d_{\hat{w}}\right] e=} \\
& d(w)^{-1}\left(i d_{a \otimes w} \otimes p_{w} \otimes i d_{\hat{w}}\right)\left(x \otimes i d_{w \otimes \hat{w}}\right)\left(i d_{a} \otimes i_{w} \otimes i_{w} p_{\hat{w}}\right)= \\
& d(w)^{-1}\left(i d_{a \otimes w} \otimes\left[\left(p_{w} \otimes i d_{\hat{w}}\right)\left(i d_{\hat{w}} \otimes i_{w} p_{\hat{w}}\right)\right]\right)\left(\left[x\left(i d_{a} \otimes i_{w}\right)\right] \otimes i d_{w \otimes \hat{w}}\right)= \\
& d(w)^{-1}\left(i d_{a \otimes w \otimes \hat{w}} \otimes p_{\hat{w}}\right)\left(\left[x\left(i d_{a} \otimes i_{w}\right)\right] \otimes i d_{w \otimes \hat{w}}\right)= \\
& d(w)^{-1}\left(x \otimes i d_{w \otimes \hat{w}}\right)\left(i d_{a} \otimes i_{w} \otimes p_{\hat{w}}\right)=x e .
\end{aligned}
$$


The Popa systems are exactly the lattices of $\mathbf{C}^{*}$-algebras (with traces) which can arise as lattices of higher relative commutants of extremal inclusions of finite index of $I I_{1}$ factors $[\mathrm{P} 4]$. There are at least two kind of examples when the Popa system $\operatorname{End}\left(v^{\otimes[i, j]}\right)_{0 \leq i \leq j<\infty}$ is the one corresponding to some "nice" subfactor associated to $(A, v)$ :

Example 2.1. (cf. WS Wet $G \rightarrow \operatorname{Aut}(P)$ be a minimal action of a compact group $G$ on a $I I_{1}$ factor $P$. If $\pi: G \rightarrow \mathcal{L}(H)$ is a finite dimensional unitary representation of $G$ then the lattice of higher relative commutants of the inclusion of fixed point algebras

$$
P^{G} \subset(P \otimes \mathcal{L}(H))^{G}
$$

is $\operatorname{End}\left(v^{\otimes[i, j]}\right)_{0 \leq i \leq j<\infty}$, where $v$ is the corepresentation corresponding to $\pi$ of the Woronowicz algebra $A=C(G)$.

Example 2.2. (cf. [P1]) Let $\Gamma \subset \operatorname{Aut}(P)$ be an outer (discrete) group of automorphisms of a $I I_{1}$ factor $P$. If $\Gamma$ is generated by $g_{1}, \ldots, g_{n}$ then the lattice of higher relative commutants of the inclusion

$$
P \subset M_{n}(P)
$$

given by $x \mapsto \operatorname{diag}\left(g_{1}(x), \ldots, g_{n}(x)\right)$ is $\operatorname{End}\left(v^{\otimes[i, j]}\right)_{0 \leq i<j<\infty}$, where $v$ is the corepresentation $\operatorname{diag}\left(u_{g_{1}}, \ldots, u_{g_{n}}\right)$ of the Woronowicz algebra $A=\mathbf{C}^{*}(\Gamma)$.

It is possible to extend the above results to coactions/actions of more general Woronowicz algebras (see for instance [PW]), and in fact this quantum group formalism shows that the above two constructions are of the same nature (i.e. one can pass from each of them to the other one by considering dual coactions/actions ; I owe this observation from E. Blanchard). In the general case Popa's universal construction is aviable:

Example 2.3. Let $\mu \in(0,1)$ and consider the fundamental representation $v$ of $\mathbf{S}_{\mu} \mathbf{U}(2)$. As $d(v)=\mu^{2}+\mu^{-2}$ and $\operatorname{End}(v) \simeq \mathbf{C}$ (see the Example 1.2), any inclusion having $\operatorname{End}\left(v^{\otimes[i, j]}\right)_{0 \leq i<j<\infty}$ as lattice of higher relative commutants has to be irreducible and of index $\lambda^{-1}=\left(\mu^{2}+\mu^{-2}\right)^{2}>4$.

The algebras $\operatorname{End}\left(v^{\otimes[i, j]}\right)$ being generated by the Jones projections (cf. the representation theory of $\mathbf{S}_{\mu} \mathbf{U}(2)$, see $[\mathbf{W}), \operatorname{End}\left(v^{\otimes[i, j]}\right)_{0 \leq i \leq j<\infty}$ is the lattice of higher relative commutants of the subfactors constructed in [P2] 


\section{Representations of Popa systems}

There are several restrictions on the Popa systems associated to corepresentations of Woronowicz algebras, for instance the index is always $\geq 4$ and the index of the amenable ones is the square of an integer (see the section 6 ). We will prove that in fact the only extra structure of a Popa system of the form $\operatorname{End}\left(v^{\otimes[i, j]}\right)_{0 \leq i \leq j<\infty}$ comes from the fact that it can be embedded into the lattice $\mathcal{L}\left(H^{\otimes[i, j]}\right)_{0 \leq i \leq j<\infty}$, where $H$ is the Hilbert space where the corepresentation $v$ acts.

Definition 3.1 A representation of a Popa system $\left(A_{i j}\right)_{0 \leq i \leq j<\infty}$ on a finite dimensional Hilbert space $H$ is a system $\pi=\left(\pi_{i j}\right)_{0 \leq i \leq j<\infty}$ of inclusions of $\mathrm{C}^{*}$-algebras $\pi_{i j}: A_{i j} \hookrightarrow \mathcal{L}\left(H^{\otimes[i, j]}\right)$ such that

$$
\pi_{k l}(x)=i d_{H \otimes[k, i]} \otimes \pi_{i j}(x) \otimes i d_{H \otimes[j, l]}
$$

for every $0 \leq k \leq i \leq j \leq l$ and $x \in A_{i j}$.

Example 3.1. If $A$ is a Woronowicz algebra and $v \in \mathcal{L}(H) \otimes A_{s}$ is a finite dimensional unitary corepresentation of $A$ then $v^{\otimes x}$ acts on $H^{\otimes x}$ for every $x \in \mathbf{N}^{* 2}$, so $\operatorname{End}\left(v^{\otimes x}\right)$ is by definition a subalgebra of $\mathcal{L}\left(H^{\otimes x}\right)$. It follows that $\operatorname{End}\left(v^{\otimes[i, j]}\right)_{0 \leq i \leq j<\infty}$ is canonically represented on $H$.

Example 3.2. Fix $\lambda \in(0,1 / 4]$ and let $\left(A_{i j}^{\lambda}\right)_{0 \leq i \leq j<\infty}$ be the Popa system generated by the $\lambda$-sequence of Jones projections (i.e. the one in the example 2.3). If $H$ is a finite dimensional Hilbert space and $Q \in \mathcal{L}(H)$ is a positive operator satisfying $\operatorname{Tr}\left(Q^{2}\right)=\operatorname{Tr}\left(Q^{-2}\right)=\lambda^{-1 / 2}$, it is easy to see that there exists a (unique) representation of $\left(A_{i j}^{\lambda}\right)_{0 \leq i \leq j<\infty}$ on $H$, sending $\pi_{2 k-2,2 k}$ : $e_{2 k} \mapsto \operatorname{Proj}_{\mathbf{C} \xi(Q)}$ and $\pi_{2 k-1,2 k+1}: e_{2 k+1} \mapsto \operatorname{Proj}_{\mathbf{C} \xi\left(\left(Q^{-1}\right)^{t}\right)}$.

In fact these are (modulo some "unitary equivalence", see below) all the representations of $\left(A_{i j}^{\lambda}\right)_{0 \leq i \leq j<\infty}$ on finite dimensional Hilbert spaces. Note that the PPTL representation is a particular case of this construction.

The second example is in fact a particular case of the first one, for $v=$ the fundamental representation of $A_{u}(Q)$. In fact the following result holds :

Theorem 3.1 For any Popa system $\left(A_{i j}\right)_{0 \leq i \leq j<\infty}$ the following conditions are equivalent :

(i) There exists a finite dimensional unitary corepresentation $v$ of a Woronowicz algebra $A$ such that $\left(A_{i j}\right)_{0 \leq i \leq j<\infty}=\operatorname{End}\left(v^{\otimes[i, j]}\right)_{0 \leq i \leq j<\infty}$. 
(ii) There exists a finite dimensional Hilbert space $H$ and a representation $\pi$ of $\left(A_{i j}\right)_{0 \leq i \leq j<\infty}$ on $H$ such that $\pi_{02}\left(e_{2}\right)$ is a rank one projection.

The implication $(i) \Longrightarrow($ ii) is clear, and the converse will be proved in the next section. In the rest of this section we give some preliminary results on the representations of Popa systems.

First of all we have to explain the meaning of the equality sign in (i). We make the following conventions : from now on by "Popa system" we mean the triple consisting of the lattice, the trace, and the sequence of Jones projections. If $v$ is a corepresentation of a Woronowicz algebra, the trace and Jones projections of the Popa system $\operatorname{End}\left(v^{\otimes[i, j]}\right)_{0 \leq i \leq j<\infty}$ are by definition the ones in the proof of the Theorem 2.1. Two Popa systems $\left(\left(A_{i j}\right)_{0 \leq i \leq j<\infty}, \tau,\left(e_{k}\right)_{k \geq 2}\right)$ and $\left(\left(A_{i j}^{\prime}\right)_{0 \leq i \leq j<\infty}, \tau^{\prime},\left(e_{k}^{\prime}\right)_{k \geq 2}\right)$ are said to be equal if there exists a system of trace-preserving, Jones projections-preserving and inclusion-preserving $\mathbf{C}^{*}$ isomorphisms $A_{i j} \rightarrow A_{i j}^{\prime}$.

Recall that the Jones projections of $\operatorname{End}\left(v^{\otimes[i, j]}\right)_{0<i<j<\infty}$ have a special form - they are the rank one projections onto $\mathbf{C} \xi\left(Q_{v}\right)$ or onto $\mathbf{C} \xi\left(\left(Q_{v}^{-1}\right)^{t}\right)$ (where $\xi: \mathcal{L}(H) \simeq H \otimes \bar{H}$ is the canonical linear map). We will need the following proposition on "unitary equivalence" of representations.

Definition 3.2 A representation $\pi$ of a Popa system $\left(A_{i j}\right)_{0 \leq i \leq j<\infty}$ on $H$ is said to be normalised if there exists a positive operator $Q \in \mathcal{L}(H)$ satisfying $\operatorname{Tr}\left(Q^{2}\right)=\operatorname{Tr}\left(Q^{-2}\right)=\lambda^{-1 / 2}$ such that $\pi_{2 k-2,2 k}\left(e_{2 k}\right)$ is the projection onto $\mathbf{C} \xi(Q)$ and $\pi_{2 k-1,2 k+1}\left(e_{2 k+1}\right)$ is the projection onto $\mathbf{C} \xi\left(\left(Q^{-1}\right)^{t}\right), \forall k \geq 1$.

Proposition 3.1 If $\pi$ is a representation such that $\pi_{02}\left(e_{2}\right)$ is a rank one projection, then there exists a sequence of unitaries $U_{1}, U_{2}, U_{3}, \ldots$ such that

$$
\pi_{i j}^{\prime}=\operatorname{ad}\left(U_{i+1} \otimes U_{i+2} \otimes \ldots \otimes U_{j}\right) \circ \pi_{i j}
$$

is normalised representation.

We will use the following elementary Lemma:

Lemma 3.1 Let $H$ be a finite dimensional Hilbert space and $e \in \mathcal{L}(H \otimes \bar{H})$, $f \in \mathcal{L}(\bar{H} \otimes H)$ be rank one projections satisfying the Jones relation

$$
\left(i d_{H} \otimes f\right)\left(e \otimes i d_{H}\right)\left(i d_{H} \otimes f\right)=\lambda\left(i d_{H} \otimes f\right)
$$

for some $\lambda>0$. If $E, F \in \mathcal{L}(H)$ are such that $\xi(E)$ and $\xi\left(F^{t}\right)$ are norm one vectors in the images of e and $f$, then $\left(F^{*} F\right)\left(E^{*} E\right)=\lambda i d_{H}$. 
Proof. If $\left\{e_{k}\right\}$ is an orthogonal basis of $H$ then a straighforward computation shows that

$$
<(i d \otimes f)(e \otimes i d)(i d \otimes f)\left(e_{a} \otimes \xi\left(F^{t}\right)\right),\left(e_{b} \otimes \xi\left(F^{t}\right)\right)>=\left(E F^{*} F E^{*}\right)_{b a}
$$

for any $a, b$, so the Jones relation implies $E F^{*} F E^{*}=\lambda I_{H}$.

Proof of the Proposition 3.1. Note firstly that the Jones relations imply (by reccurence on $n \geq 2$ ) that each $\pi_{n-2, n}\left(e_{n}\right)$ is a rank one projection.

Step I. Construction of $Q, U_{1}, U_{2}$. The above lemma applies with $e=$ $\pi_{02}\left(e_{2}\right)$ and $f=\pi_{13}\left(e_{3}\right)$. Thus if $e$ is the projection onto $\mathbf{C} \xi(E)$ then $E$ is invertible ; let $E=Q U$ be its polar decomposition. If $\left\{e_{1}, \ldots, e_{n}\right\}$ is an orthogonal basis of $H$ consisting of eigenvectors of $Q$, then $e$ is the orthogonal projection onto $\mathbf{C} \sum Q_{i i} U_{i j} e_{i} \otimes \overline{e_{j}}$. By multiplying $Q$ with a scalar, we may suppose that $\operatorname{Tr}\left(Q^{2}\right)=\operatorname{Tr}\left(Q^{-2}\right)$. We have

$$
(1 \otimes \bar{U})\left(\sum Q_{i i} U_{i j} e_{i} \otimes \overline{e_{j}}\right)=\sum Q_{i i} e_{i} \otimes \bar{e}_{i}=\xi(Q)
$$

so $\left(I_{n} \otimes \bar{U}\right) e\left(I_{n} \otimes U^{t}\right)$ is the orthogonal projection onto $\mathbf{C} \xi(Q)$. By perturbing the representation $\pi$ with the sequence of unitaries $\left\{I_{n}, \bar{U}, I_{n}, I_{n}, \ldots\right\}$ we may assume that $e=\pi_{02}\left(e_{2}\right)$ is the orthogonal projection onto $\mathbf{C} \xi(Q)$.

Step II. Construction of $U_{s}, s \geq 3$ and proof of $\operatorname{Tr}\left(Q^{2}\right)=\lambda^{-1 / 2}$. Define $e_{2 k}^{\prime}$ to be the projection onto $\mathbf{C} \xi(Q)$ and $e_{2 k+1}^{\prime}$ to be the projection onto $\mathbf{C} \xi\left(Q^{-1}\right)$, for every $k \geq 1$. We have to show that if $\pi_{k-2, k}\left(e_{k}\right)=e_{k}^{\prime}$ for $k=2,3, \ldots, s-1$ then $\left(i d \otimes \operatorname{ad}\left(U_{s}\right)\right)\left(\pi_{s-2, s}\left(e_{s}\right)\right)=e_{s}^{\prime}$ for some unitary $U_{s}$. It is enough to do it for $s=3$. Let $e=\pi_{02}\left(e_{2}\right)$ and $f=\pi_{13}\left(e_{3}\right)$ and assume that $f$ is the orthogonal projection onto $\mathbf{C} \nu$, with $\nu=\xi\left(F^{t}\right)$ a norm one vector. The above lemma shows that

$$
\left(F^{*} F\right)\left(\operatorname{Tr}\left(Q^{2}\right)^{-1} Q^{2}\right)=\lambda I_{n}
$$

and as $\nu$ is a norm one vector, $\operatorname{Tr}\left(F^{*} F\right)=1$, so $\operatorname{Tr}\left(Q^{-2}\right)=\operatorname{Tr}\left(Q^{2}\right)=\lambda^{-1 / 2}$. Also $F^{*} F=\lambda^{1 / 2} Q^{-2}$, so $F=\lambda^{1 / 4} U Q^{-1}$ for some unitary $U$. Thus $f$ is the orthogonal projection onto $\mathbf{C} \sum Q_{i i}^{-1} U_{j i} \bar{e}_{i} \otimes e_{j}$. As $\left(I_{n} \otimes U^{*}\right) \sum Q_{i i}^{-1} U_{j i} \bar{e}_{i} \otimes e_{j}=$ $\sum Q_{i i}^{-1} \bar{e}_{i} \otimes e_{i}$, one can choose $U_{3}:=U$.

Recall that the conditional expectations in a Popa system of the form $\operatorname{End}\left(v^{\otimes[i, j]}\right)_{0 \leq i \leq j<\infty}$ have a certain special form ; we will prove in the next proposition that the same happens for a Popa system having a normalised representation. 
Proposition 3.2 Let $\left(A_{i j}\right)_{0 \leq i \leq j<\infty}, H, \pi, Q$ be as in the definition 3.2. Define linear maps $i_{\alpha} \in \mathcal{L}(\mathbf{C}, H \otimes \bar{H})$ by $1 \mapsto \xi(Q)$ and $i_{\beta} \in \mathcal{L}(\mathbf{C}, \bar{H} \otimes H)$ by $1 \mapsto \xi\left(\left(Q^{-1}\right)^{t}\right)$. Let $p_{\alpha}:=i_{\beta}^{*}$ and $p_{\beta}:=i_{\alpha}^{*}$. For $x \in \mathbf{N}^{* 2}$ let $i d_{x}$ be the identity of $H^{\otimes x}$.

(i) The following "duality formulas" hold, $\forall \gamma \in\{\alpha, \beta\}$ :

$$
\left(i d_{\gamma} \otimes p_{\gamma}\right)\left(i_{\gamma} \otimes i d_{\gamma}\right)=\left(p_{\hat{\gamma}} \otimes i d_{\gamma}\right)\left(i d_{\gamma} \otimes i \hat{\gamma}\right)=i d_{\gamma}
$$

(ii) The Jones projections of $\left(A_{i j}\right)_{0 \leq i \leq j<\infty}$ are given by

$$
\pi_{2 k-2,2 k}\left(e_{2 k}\right)=\lambda^{1 / 2} i_{\alpha} p_{\beta}, \quad \pi_{2 k-1,2 k+1}\left(e_{2 k+1}\right)=\lambda^{1 / 2} i_{\beta} p_{\alpha}
$$

(iii) The horizontal conditional expectations are given by

$$
\pi_{i, j-1}\left(E_{A_{i, j-1}}(T)\right)=\lambda^{1 / 2}\left(i d_{[i, j-1]} \otimes p_{\hat{\gamma}}\right)\left(\pi_{i j}(T) \otimes i d_{\hat{\gamma}}\right)\left(i d_{[i, j-1]} \otimes i_{\gamma}\right)
$$

$\forall 0 \leq i \leq j$ and $T \in A_{i j}$, where $\gamma=\alpha$ if $j$ is odd and $\gamma=\beta$ if $j$ is even. $A$ similar formula holds for the vertical conditional expectations.

Proof. (ii) is trivial, for (i) see the proof of Prop. 1.1 (d2). For (iii) recall that by the Jones conditions the following equality holds in $A_{i, j+1}$ :

$$
E_{A_{i, j-1}}(T) e_{j+1}=e_{j+1} T e_{j+1}
$$

As $\pi_{i, j+1}\left(e_{j+1}\right)=\lambda^{1 / 2} i d_{[i, j-1]} \otimes i_{\gamma} p_{\hat{\gamma}}$, by applying $\pi_{i, j+1}$ the Jones condition becomes

$\pi_{i, j-1}\left(E_{A_{i, j-1}}(T)\right) \otimes\left(i_{\gamma} p_{\hat{\gamma}}\right)=\lambda^{1 / 2}\left(i d_{[i, j-1]} \otimes i_{\gamma} p_{\hat{\gamma}}\right)\left(\pi_{i j}(T) \otimes i d_{\hat{\gamma}}\right)\left(i d_{[i, j-1]} \otimes i_{\gamma} p_{\hat{\gamma}}\right)$

which is equal to $\lambda^{1 / 2}\left(i d_{[i, j-1]} \otimes p_{\hat{\gamma}}\right)\left(\pi_{i j}(T) \otimes i d_{\hat{\gamma}}\right)\left(i d_{[i, j-1]} \otimes i_{\gamma}\right) \otimes\left(i_{\gamma} p_{\hat{\gamma}}\right)$.

Remark. Note that only the Jones conditions were used in the above proof. In fact one can prove that any lattice $\left(A_{i j}\right)_{0 \leq i \leq j<\infty}$ of $\mathbf{C}^{*}$-algebras (with traces) which satisfies the Jones conditions and which has a normalised representation is a Popa system (the Commutation relations are trivial, the Commuting square condition is clear from the above formula for the conditional expectations, and the Markov conditions may be checked by the same computation as in the proof of the Theorem 2.1). This is the reason why in the next section we will use the Proposition 3.2 instead of Popa's four axioms. Actually, we will need not only the Prop. 3.2, but also its Corollaries 3.1 and 3.2 below. 
Corollary 3.1 If $0 \leq i \leq j$ and $T \in A_{i j}$ then

$$
\pi_{i, j-2}\left(E_{A_{i, j-2}}\left(e_{j} T e_{j}\right)\right)=\left(i d_{[i, j-2]} \otimes p_{\hat{\gamma}}\right) \pi_{i j}(T)\left(i d_{[i, j-2]} \otimes i_{\gamma}\right)
$$

where $\gamma=\beta$ if $j$ is odd and $\gamma=\alpha$ if $j$ is even.

Proof. Follows from $E_{A_{i, j-2}}=E_{A_{i, j-2}} E_{A_{i, j-1}}$ and by applying twice the Prop. 3.2. (iii).

Corollary 3.2 If $0 \leq i \leq j$ and $0 \leq k \leq l$ are such that $[i, j]=[k, l]$ in $\mathbf{N}^{* 2}$, then $\pi_{i, j}\left(A_{i, j}\right)=\pi_{k, l}\left(A_{k, l}\right)$.

Proof. By a recurrence argument, it is enough to prove it for $k=i+2$ and $l=j+2$. Recall that the canonical isomorphism $s h: A_{i j} \rightarrow A_{i+2, j+2}$ satisfies the formula (see for instance [Bi])

$$
\operatorname{sh}(T) e_{i+2}=\lambda^{i-j} e_{i+2} e_{i+3} \ldots e_{j+1} T e_{j+2} e_{j+1} \ldots e_{i+3} e_{i+2}
$$

Now by Prop. 3.2 (i,ii) we get that

$$
\begin{gathered}
\pi_{i, j+2}\left(e_{i+2} \ldots e_{j+1}\right)=\lambda^{j-i / 2} i_{\hat{\gamma}} \otimes i d_{[i, j-1]} \otimes p_{\delta} \otimes i d_{\hat{\delta}} \\
\pi_{i, j+2}\left(e_{j+2} \ldots e_{i+2}\right)=\lambda^{j-i+1 / 2} p_{\gamma} \otimes i d_{[i, j]} \otimes i_{\delta}
\end{gathered}
$$

where $\gamma, \delta \in\{\alpha, \beta\}$ depend on the parity of $i$ and $j$. Thus by applying $\pi_{i, j+2}$ to $(\star)$ we get that $\lambda^{1 / 2} i_{\hat{\gamma}} p_{\gamma} \otimes \pi_{i+2, j+2}(\operatorname{sh}(T))$ is equal to

$$
\lambda^{1 / 2} i_{\hat{\gamma}} p_{\gamma} \otimes\left(i d_{[i, j-1]} \otimes p_{\delta} \otimes i d_{\hat{\delta}}\right)\left(\pi_{i j}(T) \otimes i d_{\delta \hat{\delta}}\right)\left(i d_{[i, j]} \otimes i_{\delta}\right)
$$

so by Prop. 3.2 (i) we get $\pi_{i+2, j+2}(s h(T))=\pi_{i j}(T), \forall T \in A_{i j}$.

\section{The universal monoidal category associated with a Popa system}

We prove in this section the implication (ii) $\Longrightarrow$ (i) in the Theorem 3.1. Let $\left(A_{i j}\right)_{0 \leq i \leq j<\infty}$ be a Popa system, $H$ be a finite dimensional Hilbert space and $\pi$ be a representation of $\left(A_{i j}\right)_{0 \leq i \leq j<\infty}$ on $H$ such that $\pi_{02}\left(e_{2}\right)$ is a rank one projection. We will construct a pair $(A, u)$ satisfying (w1-3) such that 
$\left(A_{i j}\right)_{0 \leq i \leq j<\infty}=\operatorname{End}\left(u^{\otimes[i, j]}\right)_{0 \leq i \leq j<\infty}$. This pair is far from being unique (see the section 5) ; we will construct the "universal" one.

By the Proposition 3.1 we may assume that $\pi$ is normalised. Let $Q \in$ $\mathcal{L}(H)$ be as in the Definition 3.2 and define linear maps $i_{\alpha}, i_{\beta}, p_{\alpha}, p_{\beta}$ as in the Proposition 3.2.

Let $\mathbf{N}_{\text {alt }}^{* 2}$ be the subset of $\mathbf{N}^{* 2}$ consisting of alternating words, i.e. of words not containg $\alpha^{2}$ or $\beta^{2}$. Thus $\mathbf{N}_{a l t}^{* 2}=\{[i, j] \mid 0 \leq i \leq j\}$, and the Corollary 3.2 shows that to any $x \in \mathbf{N}_{\text {alt }}^{* 2}$ one can associate a $\mathbf{C}^{*}$-algebra $A_{x} \subset \mathcal{L}\left(H^{\otimes x}\right)$ by $A_{x}:=\pi_{i, j}\left(A_{i, j}\right)$ for any $0 \leq i \leq j$ such that $[i, j]=x$.

We define a monoidal subcategory $\mathcal{R}$ of the category of finite dimensional complex vector spaces in the following way. The objects of $\mathcal{R}$ are $\left\{H^{\otimes x} \mid x \in\right.$ $\left.\mathbf{N}^{* 2}\right\}$, and $\mathcal{R}$ is the smallest monoidal category containing $i_{\alpha}, i_{\beta}, p_{\alpha}, p_{\beta}$ and all the elements of all the $A_{x}$ 's, for $x \in \mathbf{N}_{\text {alt }}^{* 2}$. Equivalently, the arrows of $\mathcal{R}$ are the linear combinations of (composable) compositions of tensor products of maps of the form $i_{\alpha}, i_{\beta}, p_{\alpha}, p_{\beta}$, or of the form $i d_{x}:=i d_{H^{\otimes x}}$ with $x \in \mathbf{N}^{* 2}$, or of the form $T$ with $T \in A_{x}$ for some $x \in \mathbf{N}_{\text {alt }}^{* 2}$ (see also [W2], [B1], B2] for this kind of constructions).

By definition of $\mathcal{R}$ we have $A_{x} \subset \operatorname{End}_{\mathcal{R}}\left(H^{\otimes x}\right)$ for any $x \in \mathbf{N}_{\text {alt }}^{* 2}$. The Theorem 3.1 will follow from the reconstruction results in [W2] and from the following result.

Proposition 4.1 $\operatorname{End}_{\mathcal{R}}\left(H^{\otimes x}\right)=A_{x}$ for any $x \in \mathbf{N}_{\text {alt }}^{* 2}$.

This will be proved after the "computation" of the arrows of $\mathcal{R}$ (Lemma 4.1 and Proposition 4.2). The following special type of arrows will play an important role in this computation.

Definition 4.1 A block is a map of the form

$$
i d_{a} \otimes\left[\left(i d_{x} \otimes p_{\gamma}^{\otimes n} \otimes i d_{z}\right) T\left(i d_{y} \otimes i_{\gamma}^{\otimes m} \otimes i d_{t}\right)\right] \otimes i d_{b}
$$

where $\gamma \in\{\alpha, \beta\}, m, n \geq 0, a, b \in \mathbf{N}^{* 2}, x, y, z, t \in \mathbf{N}_{\text {alt }}^{* 2}$ and $T \in A_{w}$ for some $w \in \mathbf{N}_{\text {alt }}^{* 2}$ such that $w=x(\gamma \hat{\gamma})^{n} z=y(\gamma \hat{\gamma})^{m} t$.

The above block will be denoted $B(a, b, x, y, z, t, \gamma, n, m, w, T)$. It is an element of $H o m_{\mathcal{R}}\left(H^{\otimes a y t b}, H^{\otimes a x z b}\right)$.

Such a block is said to be connected if $a=b=e$; left degenerate if $x=z=e$; right degenerate if $y=t=e$; normalised if $z=t=e$ and $m n=0$ (where $e$ is the unit of $\mathbf{N}^{* 2}$ ). 
Lemma 4.1 Each arrow in $\mathcal{R}$ is a sum of compositions of blocks.

Proof. As the set of sums of compositions of blocks is stable by linear sums and by compositions, we may consider the category $\mathcal{R}^{\prime}$ whose objects are $\left\{H^{\otimes x} \mid x \in \mathbf{N}^{* 2}\right\}$ and whose arrows are sums of compositions of blocks. We have to show that $\mathcal{R}=\mathcal{R}^{\prime}$, and by definition of $\mathcal{R}$ and $\mathcal{R}^{\prime}$ it is enough to show that $\mathcal{R}^{\prime}$ is a monoidal category. But if $A \in \mathcal{L}\left(H^{\otimes x}, H^{\otimes y}\right)$ and $B \in$ $\mathcal{L}\left(H^{\otimes z}, H^{\otimes t}\right)$ are blocks, then $A \otimes B$ is equal to $\left(i d_{y} \otimes B\right)\left(A \otimes i d_{z}\right)$, which is a composition of blocks.

Lemma 4.2 Each block is equal to a normalised block.

Proof. We will give an explicit method for normalising a block. Let $B$ be as in the definition of blocks. By the Proposition 3.2 (i) the following formulas hold for any $\gamma \in\{\alpha, \beta\}$ :

$$
\begin{aligned}
i_{\gamma} \otimes i d_{\gamma} & =\left(i_{\gamma} p_{\hat{\gamma}} \otimes i d_{\gamma}\right)\left(i d_{\gamma} \otimes i_{\hat{\gamma}}\right) \\
p_{\gamma} \otimes i d_{\hat{\gamma}} & =\left(i d_{\hat{\gamma}} \otimes p_{\hat{\gamma}}\right)\left(i_{\hat{\gamma}} p_{\gamma} \otimes i d_{\hat{\gamma}}\right)
\end{aligned}
$$

Also by the Prop. 3.2 (ii), $i_{\gamma} p_{\hat{\gamma}}$ and $i_{\hat{\gamma}} p_{\gamma}$ are scalar multiples of Jones projections, so by multiplying $T$ to the left and to the right with suitable products of Jones projections, the $i_{\gamma}$ 's and $p_{\gamma}$ 's can be moved to the right. That is, $B$ is equal to a block $B^{\prime}$ having $z=t=e$.

If $m n=0$ we have finished. Assume $m, n \geq 1$; then $w=w^{\prime} \gamma \hat{\gamma}$ for some $w^{\prime} \in \mathbf{N}_{a l t}^{* 2}$. Thus

$$
B^{\prime}=i d_{a} \otimes\left[\left(i d_{x} \otimes p_{\hat{\gamma}}^{\otimes n-1}\right) T^{\prime}\left(i d_{y} \otimes i_{\gamma}^{\otimes m-1}\right)\right] \otimes i d_{b}
$$

with $T^{\prime}=\left(i d_{x(\gamma \hat{\gamma})^{n-1}} \otimes p_{\hat{\gamma}}\right) T\left(i d_{y(\gamma \hat{\gamma})^{n-1}} \otimes i_{\gamma}\right)$, which by the Corollary 3.1 is in $A_{w^{\prime}}$. By using this remark $|m-n|$ times, it follows that $B^{\prime}$ is equal to a normalised block.

Lemma 4.3 A product of connected blocks is a connected block.

Proof. It is enough to do it for a product $B B^{\prime}$ of two connected blocks. By the Lemma 4.2 we may assume that $B$ and $B^{\prime}$ are connected and normalised. Write

$$
B=\left(i d_{x} \otimes p_{\hat{\gamma}}^{\otimes n}\right) T\left(i d_{y} \otimes i_{\gamma}^{\otimes m}\right), \quad B^{\prime}=\left(i d_{x^{\prime}} \otimes p_{\hat{\gamma}^{\prime}}^{\otimes n^{\prime}}\right) T^{\prime}\left(i d_{y^{\prime}} \otimes i_{\gamma^{\prime}}^{\otimes m^{\prime}}\right)
$$


with $n m=n^{\prime} m^{\prime}=0$. The composability of $B$ and $B^{\prime}$ shows that $\gamma=\gamma^{\prime}$ and $y=x^{\prime}$. Now each $i_{\gamma} p_{\hat{\gamma}}$ being a scalar multiple of a Jones projection, by performing the multiplication in the middle of $B B^{\prime}$ we may assume that $m=0$ or $n^{\prime}=0$. Suppose for instance that $m=0$; then $B B^{\prime}$ is equal to

$$
\left(i d_{x} \otimes p_{\hat{\gamma}}^{\otimes n+n^{\prime}}\right)\left(T \otimes i d_{(\gamma \hat{\gamma})^{n^{\prime}}}\right) T^{\prime}\left(i d_{y^{\prime}} \otimes i_{\gamma}^{\otimes m^{\prime}}\right)
$$

As $\left(T \otimes i d_{(\gamma \hat{\gamma})^{n^{\prime}}}\right) T^{\prime} \in A_{w^{\prime}}$, it follows that $B B^{\prime}$ is a connected block.

Lemma 4.4 Let $B$ be a right non-degenerate block and $B^{\prime}$ be a left nondegenerate block. Write $B=i d_{a} \otimes A \otimes i d_{b}$ and $B^{\prime}=i d_{a^{\prime}} \otimes A^{\prime} \otimes i d_{b^{\prime}}$ with $A, A^{\prime}$ connected blocks. If $B$ and $B^{\prime}$ are composable, then $B B^{\prime}$ is a block or is equal to a map of the form $i d_{f} \otimes A \otimes i d_{g} \otimes A^{\prime} \otimes i d_{h}$, or of the form $i d_{f} \otimes A^{\prime} \otimes i d_{g} \otimes A \otimes i d_{h}$ for some $f, g, h \in \mathbf{N}^{* 2}$.

Proof. Write $B=B(a, b, x, y, z, t, \gamma, n, m, w, T)$ and $B^{\prime}=B\left(a^{\prime}, b^{\prime}, x^{\prime}, y^{\prime}\right.$, $\left.z^{\prime}, t^{\prime}, \gamma^{\prime}, n^{\prime}, m^{\prime}, w^{\prime}, T^{\prime}\right)$. As $B, B^{\prime}$ are composable we get $a y t b=a^{\prime} x^{\prime} z^{\prime} b^{\prime}$; denote by $W$ this word. By the non-degeneracy assumptions, both $y t$ and $x^{\prime} z^{\prime}$ are non-empty subwords of $W$. There are two cases :

- either $y t$ and $x^{\prime} z^{\prime}$ are disjoint subwords of $W$; in this case $B B^{\prime}$ is equal to $i d_{f} \otimes A \otimes i d_{g} \otimes A^{\prime} \otimes i d_{h}$ (if $y t$ is at the right of $x^{\prime} z^{\prime}$ ) or to $i d_{f} \otimes A^{\prime} \otimes i d_{g} \otimes A \otimes i d_{h}$ (if $y t$ is at the left of $x^{\prime} z^{\prime}$ ) for some $f, g, h \in \mathbf{N}^{* 2}$.

- either $y t$ and $x^{\prime} z^{\prime}$ have at least one common letter as subwords of $W$; we will prove in this case that $B B^{\prime}$ is a block. As $w=y(\gamma \hat{\gamma})^{m} t$ and $w^{\prime}=$ $x^{\prime}\left(\gamma^{\prime} \hat{\gamma}^{\prime}\right)^{n^{\prime}} t^{\prime}$ are alternating words, it follows that $y t$ and $x^{\prime} z^{\prime}$ are alternating words, so their union in $W$ is an alternating word, say $V$. Write $W=a^{\prime \prime} V b^{\prime \prime}$ ; then $a^{\prime \prime}$ is a subword of $a$, and if $\varepsilon$ is such that $a=a^{\prime \prime} \varepsilon$ it follows that $\varepsilon x \in \mathbf{N}_{a l t}^{* 2}$. Thus

$$
B=i d_{a^{\prime \prime}} \otimes\left[\left(i d_{\varepsilon x} \otimes p_{\hat{\gamma}}^{\otimes n} \otimes i d_{z}\right)\left(i d_{\varepsilon} \otimes T\right)\left(i d_{y} \otimes i_{\gamma}^{\otimes m} \otimes i d_{t}\right)\right] \otimes i d_{b}
$$

with $i d_{\varepsilon} \otimes T \in A_{\varepsilon w}$, so we may suppose that $a=a^{\prime \prime}$. By the same argument we may suppose $a^{\prime}=a^{\prime \prime}$, and also $b=b^{\prime \prime}$ and $b^{\prime}=b^{\prime \prime}$. In this way $B B^{\prime}$ becomes of the form $i d_{a^{\prime \prime}} \otimes K \otimes i d_{b^{\prime \prime}}$, with $K$ a product of connected blocks. By the Lemma $4.3 K$ is a connected block, so $B B^{\prime}$ is a block.

Proposition 4.2 Every composition of blocks is of the form

$$
C_{1} \ldots C_{n} B_{1} \ldots B_{m} A_{1} \ldots A_{p}
$$

where the $A_{i}$ 's are left degenerate blocks, the $C_{i}$ 's are right degenerate blocks, and the $B_{i}$ 's are left and right non-degenerate blocks. 
Proof. Let us call $\mathcal{A}$ (resp. $\mathcal{C}, \mathcal{B}$ ) the set of left degenerate (resp. right degenerate, left and right non-degenerate) blocks. The Lemma 4.4 shows that:

- if $A \in \mathcal{A}$ and $C \in \mathcal{C}$ then $A C$ is a block or is of the form $C^{\prime} A^{\prime}$, with $A^{\prime} \in \mathcal{A}$ and $C^{\prime} \in \mathcal{C}$.

- if $A \in \mathcal{A}$ and $B \in \mathcal{B}$ then $A B$ is a block or is of the form $B^{\prime} A^{\prime}$, with $A^{\prime} \in \mathcal{A}$ and $B^{\prime} \in \mathcal{R}$.

- if $B \in \mathcal{B}$ and $C \in \mathcal{C}$ then $B C$ is a block or is of the form $C^{\prime} B^{\prime}$, with $B^{\prime} \in \mathcal{B}$ and $C^{\prime} \in \mathcal{C}$.

Let $X$ be a composition of blocks, and choose a decomposition of $X$ as product of minimal number of blocks. By performing the above operations one may write $X$ as in the statement.

Remark. The Lemma 4.1 and the Proposition 4.2 give the structure of arrows in $\mathcal{R}$, as "reduced words on blocks". Some better statements may be proved ; however, there is no simple writing for a product of type $A_{1} \ldots A_{p}$ or $C_{1} \ldots C_{n}$.

Lemma 4.5 (i) Let $B \in H_{\mathcal{O}}\left(H^{\otimes u}, H^{\otimes v}\right)$ be a right non-degenerate block. If $u \in \mathbf{N}_{\text {alt }}^{* 2}$ then $v \in \mathbf{N}_{\text {alt }}^{* 2}$ and $B$ is equal to a connected block.

(ii) Let $B \in H_{o m}\left(H^{\otimes u}, H^{\otimes v}\right)$ be a left non-degenerate block. If $v \in \mathbf{N}_{\text {alt }}^{* 2}$ then $u \in \mathbf{N}_{\text {alt }}^{* 2}$ and $B$ is equal to a connected block.

Proof. We prove only (i). Write $B=B(a, b, x, y, z, t, \gamma, n, m, w, T)$. Then $w=x(\gamma \hat{\gamma})^{n} z=y(\gamma \hat{\gamma})^{m} t$ (see the definition of blocks), $y t \neq e$ (by nondegeneracy) and $u=a y t b, v=a x z b\left(\right.$ as $\left.B \in H_{o m}\left(H^{\otimes u}, H^{\otimes v}\right)\right)$. As $u=$ $a y t b$ is an alternating word, it follows that:

- $a, b$ are alternating words.

- if $a$ ends with $\alpha$ (resp. $\beta$ ) then $y t$ begins with $\beta$ (resp. $\alpha$ ).

- if $b$ begins with $\alpha$ (resp. $\beta$ ) then $y t$ ends with $\beta$ (resp. $\alpha$ ).

Also as $w=y(\gamma \hat{\gamma})^{m} t$, the alternating words $w$ and $y t$ begin (resp. end) with the same letter. It follows that $a w b$ is an alternating word, and as $v$ is obtained from $a w b$ by deleting some $\gamma \hat{\gamma}$ 's, it is an alternating word. Also $a w b \in \mathbf{N}_{\text {alt }}^{* 2}$ shows that $B$ is equal to the connected block

$$
\left(i d_{a x} \otimes p_{\hat{\gamma}}^{\otimes n} \otimes i d_{z b}\right)\left(i d_{a} \otimes T \otimes i d_{b}\right)\left(i d_{a y} \otimes i_{\gamma}^{\otimes m} \otimes i d_{t b}\right)
$$


Lemma 4.6 If $x, y \in \mathbf{N}_{\text {alt }}^{* 2}$ then the elements $\operatorname{Hom}_{\mathcal{R}}\left(H^{\otimes x}, H^{\otimes y}\right)$ are sums of connected blocks.

Proof. Let $X \in H_{0} m_{\mathcal{R}}\left(H^{\otimes x}, H^{\otimes y}\right)$. By the Lemma 4.1 we may assume that $X$ is a composition of blocks. Write

$$
X=C_{1} \ldots C_{n} B_{1} \ldots B_{m} A_{1} \ldots A_{p}
$$

as in the Prop. 4.2. As all $A_{i}$ 's are left degenerate, we may assume that they are right non-degenerate (a block which is both left and right degenerate is a scalar). Thus the Lemma 4.5 (i) applies $p+m$ times and shows reccursively that $A_{p}, \ldots, A_{1}, B_{m}, \ldots, B_{1}$ are connected blocks. Also Lemma 4.5 (ii) applies $n+m$ times and shows reccursively that $C_{1}, \ldots, C_{n}, B_{1}, \ldots, B_{m}$ are connected blocks. Thus $X$ is a composition of connected blocks, and by the Lemma 4.3 it is a connected block.

Proof of the Proposition 4.1. The Lemma 4.5 shows that every element $X \in \operatorname{End}_{\mathcal{R}}\left(H^{\otimes x}\right)$ is a sum of connected blocks. By the Lemma 4.2, $X$ is a sum of normalised connected blocks. But the only normalised connected blocks in $E n d_{\mathcal{R}}\left(H^{\otimes x}\right)$ are the elements of $A_{x}$ (see the definition of blocks), so we get $E n d_{\mathcal{R}}\left(H^{\otimes x}\right) \subset A_{x}$. The other inclusion is clear by construction of $\mathcal{R}$.

End of the proof of (ii) $\Longrightarrow$ (i) in the Theorem 3.1. We use here freely the terminology from [W2]. Let us view $\mathcal{R}$ as a concrete monoidal (uncomplete) $\mathbf{W}^{*}$-category. The duality formulas in the Prop. 3.2 (i) show that the objects $H$ and $\bar{H}$ are conjugate in $\mathcal{R}$. Thus the Theorem 1.3. in W2 applies and shows that the $\mathcal{R}$-universal admissible pair $(A, u)$ is a compact matrix pseudogroup (i.e. it satisfies the conditions (w1-3) from the first section). Let $w \in \mathcal{L}(\bar{H}) \otimes A_{s}$ be the corepresentation corresponding to the object $\bar{H}$. By the next lemma $w=\hat{u}$, so the Theorem 1.3 in W2 shows also that the space of intertwiners $\operatorname{Hom}\left(u^{\otimes x}, u^{\otimes y}\right)$ is equal (as a subspace of $\mathcal{L}\left(H^{\otimes x}, H^{\otimes y}\right)$ ) to the space $H_{o m}\left(H^{\otimes x}, H^{\otimes y}\right)$ of arrows of $\mathcal{R}$, for any $x, y \in \mathbf{N}^{* 2}$. In particular if $0 \leq i \leq j$ then

$$
\operatorname{End}\left(u^{\otimes[i, j]}\right)=\operatorname{End}_{\mathcal{R}}\left(H^{\otimes[i, j]}\right)=A_{[i, j]}=\pi_{i, j}\left(A_{i, j}\right)
$$

By the next Lemma $Q_{u}=Q$, so the Jones projections and the conditional expectations of $\left(A_{i j}\right)_{0 \leq i \leq j<\infty}$ and of $\operatorname{End}\left(v^{\otimes[i, j]}\right)_{0 \leq i \leq j<\infty}$ are given by the same formulas (cf. Prop. 3.2 (ii,iii) and the proof of the Theorem 2.1). Thus 
the above identifications $\operatorname{End}\left(u^{\otimes[i, j]}\right) \simeq A_{i, j}$ are Jones projections-preserving and trace-preserving (the traces being particular cases of conditional expectations).

Lemma $4.7 w=\hat{u}$ and $Q_{u}=Q$.

Proof. We firstly prove that $w=U \hat{u} U^{*}$ for some unitary $U$. We know from $\mathrm{W} 2$ that $w$ is equivalent to $\hat{u}$. Let $F \in M_{n}(\mathbf{C})$ be such that $w=$ $F \hat{u} F^{-1}$. By polar decomposition, we may assume that $F$ is positive and we have to prove that $w=\hat{u}$ in this case. As $w$ and $u$ are unitaries

$$
w=((i d \otimes \kappa) w)^{*}=\left(F(i d \otimes \kappa)(\hat{u}) F^{-1}\right)^{*}=F^{-1}((i d \otimes \kappa) \hat{u})^{*} F=F^{-1} \hat{u} F
$$

It follows that $F^{2} \in \operatorname{End}(\hat{u})$, so $F \in \operatorname{End}(\hat{u})$ and $w=F \hat{u} F^{-1}=\hat{u}$ as desired.

As $i_{\alpha} \in \operatorname{Hom}(1, u \otimes w)$ and $i_{\beta} \in \operatorname{Hom}(1, w \otimes u)$, it is easy to see that $w=Q^{t} \bar{u}\left(Q^{-1}\right)^{t}$. On the other hand $w=U \hat{u} U^{*}$ and $\bar{u}=\left(Q_{u}^{-1}\right)^{t} \hat{u} Q_{u}^{t}$, so $U^{*} Q^{t}\left(Q_{u}^{-1}\right)^{t} \in \operatorname{End}(\hat{u})$. By polar decomposition both $U^{*}$ and $Q^{t}\left(Q_{u}^{-1}\right)^{t}$ are in $\operatorname{End}(\hat{u})$. As $U^{*} \in \operatorname{End}(\hat{u})$, we get the first assertion $w=U \hat{u} U^{*}=\hat{u}$. The other relation $Q^{t}\left(Q_{u}^{-1}\right)^{t} \in \operatorname{End}(\hat{u})$ shows that

$$
\left[Q_{u}^{t} \bar{u}\left(Q_{u}^{-1}\right)^{t}\right] Q^{t}\left(Q_{u}^{-1}\right)^{t}=Q^{t}\left(Q_{u}^{-1}\right)^{t}\left[Q_{u}^{t} \bar{u}\left(Q_{u}^{-1}\right)^{t}\right]
$$

It follows that $\left(Q Q_{u}^{-1}\right)^{t} \in \operatorname{End}(\bar{u})$, so $Q Q_{u}^{-1} \in \operatorname{End}(u)$. Thus the condition (iv) of the Lemma 1.2 is satisfied. Also $Q$ is positive, so for proving $Q=Q_{u}$ it is enough to show that $\operatorname{Tr}\left(Q^{-2}\right.$. $)=\operatorname{Tr}\left(Q^{2}\right.$.) on $\operatorname{End}(u)$. Indeed, the Proposition 3.2 shows that the expectation $\pi_{01}\left(A_{01}\right) \rightarrow \pi_{00}\left(A_{00}\right)=\mathbf{C}$ is $\operatorname{Tr}\left(Q^{2}\right.$.), and that the expectation $\pi_{01}\left(A_{01}\right) \rightarrow \pi_{11}\left(A_{11}\right)=\mathbf{C}$ is $\operatorname{Tr}\left(Q^{-2}\right.$.). As both expectations coincide with the trace on $\pi_{01}\left(A_{01}\right)$, and as $\pi_{01}\left(A_{01}\right)=$ $\operatorname{End}(u)$, we get $\operatorname{Tr}\left(Q^{-2}.\right)=\operatorname{Tr}\left(Q^{2}\right.$.) on $\operatorname{End}(u)$.

\section{From Woronowicz algebras to Popa sys- tems and back}

Let $X$ be the set of pairs (Woronowicz algebra, corepresentation) and $Y$ be the set of pairs (Popa system, normalised representation). In the Theorem 2.1 we constructed a map $L: X \rightarrow Y$ by

$$
L(A, u)=\left(\operatorname{End}\left(u^{\otimes[i, j]}\right)_{0 \leq i \leq j<\infty}, \pi\right)
$$


where $\pi$ is the canonical representation (see the Example 3.1). The Theorem 3.1 says that $L$ is surjective. Moreover, the proof of the Theorem 3.1 was as follows - to any $a \in Y$ we have associated a certain category $\mathcal{R}$; then the universal $\mathcal{R}$-admissible pair $R(a):=(A, u)$ was shown to satisfy $L(A, u)=a$. The map $R: Y \rightarrow X$ being a section for $L$, it follows that the composition

$$
R L: X \rightarrow X
$$

is a projection. We give in this section an explicit description of $R L$.

First of all we will give more precise definitions for $X$ and $Y$. We begin with $X$. For simplicity we restrict attention to the pairs $(A, u)$ satisfying the conditions $(w 1-3)$ in the first section. We will use the following special type of morphisms :

Definition 5.1 If $(A, u)$ and $(B, v)$ satisfy (w1-3), a strong morphism from $(A, u)$ to $(B, v)$ is a *-algebra morphism $f: A_{s} \rightarrow B_{s}$ such that $(i d \otimes f) u=v$.

This definition has to be understood as follows. If $u \in M_{n}\left(A_{s}\right)$ and $v \in M_{m}\left(B_{s}\right)$ with $m \neq n$ there is no strong morphism between $(A, u)$ and $(B, v)$. If $m=n$ then there exists at most one strong morphism, which has to send $u_{i j} \mapsto v_{i j}$ for any $1 \leq i, j \leq n$. Example : if $G=<g_{1}, \ldots, g_{n}>$ and $H=<h_{1}, \ldots, h_{m}>$ are finitely generated discrete groups, there exists a strong morphism

$$
\left(\mathbf{C}^{*}(G), \operatorname{diag}\left(u_{g_{1}}, \ldots, u_{g_{n}}\right)\right) \rightarrow\left(\mathbf{C}^{*}(H), \operatorname{diag}\left(u_{h_{1}}, \ldots, u_{h_{m}}\right)\right)
$$

iff $m=n$ and there exists a group morphism $G \rightarrow H$ sending $g_{i} \mapsto h_{i}$ for every $i$. Note also that $\mathbf{C}^{*}(G)$ and $\mathbf{C}_{r e d}^{*}(G)$ are strongly isomorphic ; more generally, given any $(A, u)$, if $A_{p}$ and $A_{\text {red }}$ are the full and reduced version of $A$, then $(A, u),\left(A_{p}, u\right),\left(A_{\text {red }}, u\right)$ are strongly isomorphic (see [W1, [BS]).

The following consequence of the uniqueness of strong morphisms will be used several times : if $f:(A, u) \rightarrow(B, v)$ and $g:(B, v) \rightarrow(A, u)$ are strong morphisms, then $f$ and $g$ are both strong isomorphisms.

We define $X$ to be the category of pairs $(A, u)$ satisfying (w1-3), with the strong morphisms. $Y$ is by definition the set of quadruples consisting of a Popa system $\left(A_{i j}\right)_{0 \leq i \leq j<\infty}$, a Hilbert space $H$, a positive operator $Q \in \mathcal{L}(H)$, and a representation $\pi$ of $\left(A_{i j}\right)_{0 \leq i \leq j<\infty}$ on $H$ as in the Definition 3.2, with the obvious notion of equality for such quadruples. Let us firstly give the abstract description of the map $R L$. 
Proposition 5.1 To any pair $(A, u)$ satisfying (w1-3) we associate a category $\mathcal{C}(A, u)$ in the following way:

- the objects of $\mathcal{C}(A, u)$ are the pairs $(B, v)$ satisfying (w1-3) and such that $L(B, v)=L(A, u)$.

- the arrows of $\mathcal{C}(A, u)$ are the strong morphisms.

Then $R L(A, u)$ is the (unique) universally repelling object of $\mathcal{C}(A, u)$.

Proof. Consider the Popa system $\operatorname{End}\left(u^{\otimes[i, j]}\right)_{0 \leq i \leq j<\infty}$ together with its canonical normalised representation $\pi$. Let $\mathcal{R}$ be the category defined in the fourth section, so that $R L(A, u)$ is the universal $\mathcal{R}$-admissible pair. By definition of $\mathcal{R}$ and of $\mathcal{C}(A, u)$ we see that every element of $\mathcal{C}(A, u)$ is an $\mathcal{R}$-admissible pair, so the assertion is just a traduction of the universal property of the universal admissible pair (the unicity up to strong isomorphism is clear from the unicity of strong morphisms).

We will need the following results on the free products of discrete quantum groups [Wn]. If $A$ and $B$ are Woronowicz algebras, so is their free product $A * B$ (= coproduct in the category of unital $\mathbf{C}^{*}$-algebras). $\operatorname{Irr}(A * B)-\{1\}$ is then the set of alternating products of elements of $\operatorname{Irr}(A)-\{1\}$ with elements of $\operatorname{Irr}(B)-\{1\}$. The Haar measure of $A * B$ is the free product $h * k$ of Haar measures $h$ of $A$ and $k$ of $B$. If $*_{\text {red }}$ denotes the reduced free product with respect to $h * k$, then $A *_{\text {red }} B$ is also a Woronowicz algebra (which is equal to the reduced version of the Woronowicz algebra $A * B$ ). The $\mathbf{C}^{*}$-algebras $A_{\text {red }}$ and $B_{\text {red }}$ are embedded in $A *_{r e d} B$, and are free in the sense of VDN with respect to $h * k$.

Consider the Woronowicz algebra $\mathbf{C}^{*}(\mathbf{Z})$ and let $z$ be the unitary of $\mathbf{C}^{*}(\mathbf{Z})$ corresponding to the generator 1 of $\mathbf{Z}$; it is a one-dimensional corepresentation of $\mathbf{C}^{*}(\mathbf{Z})$. Recall that by Pontrjagyn duality we have a canonical isomorphism $\mathbf{C}^{*}(\mathbf{Z}) \simeq C(\mathbf{T})$, which maps $z$ to the function $\mathbf{T} \ni x \mapsto x \in \mathbf{C}$.

Theorem 5.1 To any pair $(A, u)$ satisfying (w1-3) we associate a pair $(\tilde{A}, \tilde{u})$ satisfying (w1-3) in the following way: $\tilde{A}$ is the $\mathbf{C}^{*}$-subalgebra of $\mathbf{C}^{*}(\mathbf{Z}) * A$ generated by the entries of the matrix $\tilde{u}:=z u$. Then $(\tilde{A}, \tilde{u})$ is (strongly isomorphic to) the universally repelling object $R L(A, u)$ of $\mathcal{C}(A, u)$.

We begin with a few remarks on the operation $(A, u) \mapsto(\tilde{A}, \tilde{u})$. Firstly, as $\tilde{u}$ is a corepresentation of $\mathbf{C}^{*}(\mathbf{Z}) * A$, the comultiplication and the antipode of $\tilde{A}$ are the restrictions of the ones of $\mathbf{C}^{*}(\mathbf{Z}) * A$. Note however that the 
$\mathbf{C}^{*}$-algebra $\tilde{A}$ depends on both $u$ of $A$. The reduced version $\tilde{A}_{\text {red }}$ is the $\mathbf{C}^{*}$ subalgebra of $\mathbf{C}^{*}(\mathbf{Z}) *_{\text {red }} A$ generated by the entries of the matrix $z u$. Note that the full version $\tilde{A}_{p}$ may be different from $\tilde{A}$. Of course, $\left(\tilde{A}_{p}, \tilde{u}\right),(\tilde{A}, \tilde{u})$ and $\left(\tilde{A}_{\text {red }}, \tilde{u}\right)$ are strongly isomorphic.

Lemma 5.1 (i) $(A, u) \mapsto(\tilde{A}, \tilde{u})$ is functorial.

(ii) There exists a strong morphism $(\tilde{A}, \tilde{u}) \rightarrow(A, u)$.

(iii) $(\tilde{\tilde{A}}, \tilde{\tilde{u}})$ and $(\tilde{A}, \tilde{u})$ are strongly isomorphic.

Proof. As there is at most one strong morphism between two objects, the point (i) states precisely that if $(A, u)$ and $(B, v)$ are such that there exists a strong morphism $f:(A, u) \rightarrow(B, v)$, then there exists a strong morphism $(\tilde{A}, \tilde{u}) \rightarrow(\tilde{B}, \tilde{v})$. Such a strong morphism $\tilde{f}$ may be constructed in the following way : $\tilde{f}$ is the restriction to $\tilde{A}_{s}$ of the $\mathbf{C}^{*}$-morphism $\psi$ : $\mathbf{C}^{*}(\mathbf{Z}) * A \rightarrow \mathbf{C}^{*}(\mathbf{Z}) * B$ defined by $\left.\psi\right|_{\mathbf{C}^{*}(\mathbf{Z})}=i d$ and $\left.\psi\right|_{A}=f$.

A strong morphism as in (ii) could be constructed as the restriction to $\tilde{A}_{s}$ of the $\mathbf{C}^{*}$-morphism $\psi: \mathbf{C}^{*}(\mathbf{Z}) * A \rightarrow A$ defined by $\left.\psi\right|_{\mathbf{C}^{*}(\mathbf{Z})}=\left.\varepsilon\right|_{\mathbf{C}^{*}(\mathbf{Z})}$ (the counit of $\left.\mathbf{C}^{*}(\mathbf{Z})\right)$ and $\left.\psi\right|_{A}=i d_{A}$.

For (iii) note that $\tilde{\tilde{A}}_{s}$ is the $*$-subalgebra of $\mathbf{C}^{*}(\mathbf{Z}) * \mathbf{C}^{*}(\mathbf{Z}) * A$ generated by the entries of $\tilde{\tilde{u}}=z^{\prime} z u$, so a strong morphism $(\tilde{A}, \tilde{u}) \rightarrow(\tilde{\tilde{A}}, \tilde{\tilde{u}})$ could be constructed as the restriction to $\tilde{A}_{s}$ of the $\mathbf{C}^{*}$-morphism $\phi: \mathbf{C}^{*}(\mathbf{Z}) * A \rightarrow$ $\mathbf{C}^{*}(\mathbf{Z}) * \mathbf{C}^{*}(\mathbf{Z}) * A$ defined by $\left.\phi\right|_{\mathbf{C}^{*}(\mathbf{Z})}=\left.z^{\prime} z \varepsilon\right|_{\mathbf{C}^{*}(\mathbf{Z})}$ and $\left.\phi\right|_{A}=i d_{A}$. Now (iii) follows from (ii) and from the unicity of the strong morphisms.

We have to prove that the functor $(A, u) \mapsto(\tilde{A}, \tilde{u})$ "keeps fixed the associated Popa system" and "destroys the rest of the structure". The first assertion is easy and is the next Lemma ; the other assertion will follow from the Prop. 5.1 and from the isomorphism criterion Prop. 5.2.

Lemma 5.2 $Q_{\tilde{u}}=Q_{u}, \widehat{\tilde{u}}=\hat{u} z^{*}$, and $L(\tilde{A}, \tilde{u})=L(A, u)$.

Proof. The first two assertions are clear from the Lemma 1.2. Now using $\tilde{u}=z u, \widehat{\tilde{u}}=\hat{u} z^{*}$ and $z z^{*}=z^{*} z=1$ we find that for every $x \in \mathbf{N}_{a l t}^{* 2}$,

$$
\tilde{u}^{\otimes x}=z^{m} u^{\otimes x} z^{n}
$$

for some $m, n \in\{-1,0,1\}$. Thus $\operatorname{End}\left(\tilde{u}^{\otimes x}\right)=\operatorname{End}\left(u^{\otimes x}\right)$ for any alternating word $x$, and this proves $L(\tilde{A}, \tilde{u})=L(A, u)$. 
Lemma 5.3 Let $f:(A, u) \rightarrow(B, v)$ be a strong morphism. If

$$
\operatorname{dim}\left(\operatorname{Hom}\left(u^{\otimes x}, u^{\otimes y}\right)\right)=\operatorname{dim}\left(\operatorname{Hom}\left(v^{\otimes x}, v^{\otimes y}\right)\right)
$$

for any $x, y \in \mathbf{N}^{* 2}$, then $f$ is a strong isomorphism.

Proof. Let $f_{*}$ be the map induced by $f$ at the level of classes of corepresentations. Let $y$ be an arbitrary element of $\mathbf{N}^{* 2}$. Write $u^{\otimes y}=\sum m_{i} r_{i}$, with $m_{i}>0$ and $r_{i}$ irreducible and disjoint. Then

$$
v^{\otimes y}=\left(f_{*} u\right)^{\otimes y}=f_{*}\left(u^{\otimes y}\right)=\sum m_{i} f_{*}\left(r_{i}\right)
$$

By W1 we have $\operatorname{dim}\left(\operatorname{End}\left(u^{\otimes y}\right)\right)=\sum m_{i}^{2}$, and $\operatorname{dim}\left(\operatorname{End}\left(v^{\otimes y}\right)\right) \geq \sum m_{i}^{2}$, with equality iff the $f_{*}\left(r_{i}\right)^{\prime}$ 's are irreducible and disjoint. As $\operatorname{dim}\left(\operatorname{End}\left(u^{\otimes y}\right)\right)=$ $\operatorname{dim}\left(\operatorname{End}\left(v^{\otimes y}\right)\right)$, the $f_{*}\left(r_{i}\right)$ 's have to be irreducible.

Also from $\operatorname{dim}\left(\operatorname{Hom}\left(1, u^{\otimes y}\right)\right)=\operatorname{dim}\left(\operatorname{Hom}\left(1, v^{\otimes y}\right)\right)$ we get that $r_{i} \neq 1$ implies $f_{*}\left(r_{i}\right) \neq 1$. As every irreducible corepresentation of $A$ appears in some tensor product of the form $u^{\otimes y}$ (see [W1]) we find that $f_{*}$ maps $\operatorname{Irr}(A)-\{1\}$ into $\operatorname{Irr}(B)-\{1\}$.

Now let $a \in A_{s}$ be arbitrary. Write $a=\lambda 1+\sum a_{i}$, with $\lambda \in \mathbf{C}$ and with $a_{i}=$ coefficients of irreducible non-trivial corepresentations of $A$ (cf. WW1]). Then $f(a)=\lambda 1+\sum f\left(a_{i}\right)$, and by the above result the $f\left(a_{i}\right)$ 's are coefficients of irreducible non-trivial corepresentations of $B$. Now if $h_{A}, h_{B}$ are the Haar measures of $A$ and $B$, it follows that $h_{B}(f(a))=\lambda=h_{A}(a)$. Thus the equality $h_{B} f=h_{A}$ holds on $A_{s}$, and by positivity we get that the restriction of $f$ to $A_{s}$ is injective.

Proposition 5.2 Let $f:(A, u) \rightarrow(B, v)$ be a strong morphism. If

$$
\operatorname{dim}\left(\operatorname{End}\left(u^{\otimes x}\right)\right)=\operatorname{dim}\left(\operatorname{End}\left(v^{\otimes x}\right)\right)
$$

for any $x \in \mathbf{N}_{\text {alt }}^{* 2}$, then $(\tilde{A}, \tilde{u})$ and $(\tilde{B}, \tilde{v})$ are strongly isomorphic.

Proof. By functoriality (Lemma 5.1. (i)), the conclusion will follow from the Lemma 5.3 once we will prove that

$$
\operatorname{dim}\left(\operatorname{Hom}\left(\tilde{u}^{\otimes x}, \tilde{u}^{\otimes y}\right)\right)=\operatorname{dim}\left(\operatorname{Hom}\left(\tilde{v}^{\otimes x}, \tilde{v}^{\otimes y}\right)\right)
$$

for any $x, y \in \mathbf{N}^{* 2}$. In turn, this is equivalent to the following statement: 
If $(A, u)$ satisfies (w1-3) then the numbers $\operatorname{dim}\left(\operatorname{Hom}\left(\tilde{u}^{\otimes x}, \tilde{u}^{\otimes y}\right)\right)$ (with $\left.x, y \in \mathbf{N}^{* 2}\right)$ depend only on the numbers $\operatorname{dim}\left(\operatorname{End}\left(u^{\otimes x}\right)\right)\left(\right.$ with $\left.x \in \mathbf{N}_{a l t}^{* 2}\right)$.

We will use the following key remark (see also [B2]). If $(B, v)$ satisfies (w1-3) then by the orthogonality formulas for characters in [W1] we have

$$
\operatorname{dim}\left(\operatorname{Hom}\left(v^{\otimes x}, v^{\otimes y}\right)\right)=h \chi\left(v^{\otimes x \bar{y}}\right)=h\left(\chi(v)^{x \bar{y}}\right) \quad(\star)
$$

where $h \in B^{*}$ is the Haar measure and $\chi$ is the character of corepresentations (and where $\chi(v)^{z}$ denotes the image of $z \in \mathbf{N}^{* 2}$ by the morphism of involutive monoids $\mathbf{N}^{* 2} \rightarrow(B, \cdot, *)$ defined by $\left.\alpha \mapsto \chi(v)\right)$. Thus the numbers $(\star)$ are the $*$-moments of the non commutative random variable $\chi(v) \in(B, h)$ (in the sense of $\mathrm{VDN})$.

By [Wn, $z$ and $\chi(u)$ are $*$-free in $\mathbf{C}^{*}(\mathbf{Z}) * A$ with respect to its Haar measure, so $\left(\chi(\tilde{u}), \chi(\tilde{u})^{*}\right)=\left(z \chi(u), \chi(u)^{*} z^{*}\right)$ is an $R$-diagonal pair in the sense of [NS]. By [NS], formula (1.12) the determining series of the $R$ transform of $\left(\chi(\tilde{u}), \chi(\tilde{u})^{*}\right)$ depends only on the $R$-transform of $\chi(u) \chi(u)^{*}$. This means that the $*$-moments of $\chi(\tilde{u})$ depend only on the moments of $\chi(u) \chi(u)^{*}$. But the above remark shows that the *-moments of $\chi(\tilde{u})$ are the numbers $\operatorname{dim}\left(\operatorname{Hom}\left(\tilde{u}^{\otimes x}, \tilde{u}^{\otimes y}\right)\right.$ ) (with $x, y \in \mathbf{N}^{* 2}$ ), and that the moments of $\chi(u) \chi(u)^{*}$ are the numbers $\operatorname{dim}\left(\operatorname{End}\left(u^{\otimes x}\right)\right)$ with $x \in \mathbf{N}_{\text {alt }}^{* 2}$.

Proof of the Theorem 5.1. Let $(B, v)$ be the universally repelling object of $\mathcal{C}(A, u)$. By its universal property we get a strong morphism $(B, v) \rightarrow(A, u)$, and as $L(B, v)=L(A, u)$, the Prop. 5.2 applies and shows that $(\tilde{A}, \tilde{u})$ and $(\tilde{B}, \tilde{v})$ are strongly isomorphic.

The Lemma 5.2 shows that $(\tilde{B}, \tilde{v}) \in \mathcal{C}(A, u)$, so by the universal property of $(B, v)$ we get a strong morphism $(B, v) \rightarrow(\tilde{B}, \tilde{v})$. Together with the Lemma 5.1 (ii) and with the unicity of strong morphisms, this shows that $(B, v)$ and $(\tilde{B}, \tilde{v})$ are strongly isomorphic.

The operation $(A, u) \mapsto(\tilde{A}, \tilde{u})$ seems to be interesting, and we end this section with a complete computation for discrete groups, and with a few remarks, to be proved somewhere else.

Proposition 5.3 If $\Gamma$ is a discrete group generated by $g_{1}, \ldots, g_{n}$ then

$$
\left(\tilde{\mathbf{C}}^{*}(\Gamma), \tilde{\operatorname{diag}}\left(u_{g_{1}}, \ldots, u_{g_{n}}\right)\right) \simeq\left(\mathbf{C}^{*}(\tilde{\Gamma}), \operatorname{diag}\left(u_{z g_{1}}, \ldots, u_{z g_{n}}\right)\right)
$$

where $\tilde{\Gamma}$ is the subgroup of $\mathbf{Z} * \Gamma$ generated by $z g_{1}, \ldots, z g_{n}$ and $z$ is the generator of $\mathbf{Z}$. The group $\tilde{\Gamma}$ could be computed as follows - if $H$ is the subgroup of $\Gamma$ generated by $\left\{g_{i}^{-1} g_{j} \mid i, j=1 \ldots n\right\}$ then: 
(i) if $H=\Gamma$ then $\tilde{\Gamma}=\mathbf{Z} * \Gamma$.

(ii) if $H \neq \Gamma$ then there exists an isomorphism $\tilde{\Gamma} \rightarrow \mathbf{Z} * H$ sending $z g_{1} \mapsto z$ and $z g_{i} \mapsto g_{1}^{-1} g_{i}$ for $i=2, \ldots, n$.

Proof. As $\tilde{\Gamma}=<z g_{1},\left(z g_{1}\right)\left(g_{1}^{-1} g_{2}\right), \ldots,\left(z g_{1}\right)\left(g_{1}^{-1} g_{n}\right)>=<z g_{1}, H>$ there are two cases :

(i) if $H=\Gamma$ then $g_{1} \in H$, so $z \in \tilde{\Gamma}$, so $\tilde{\Gamma}=\mathbf{Z} * \Gamma$.

(ii) if $H \neq \Gamma$ then $g_{1} \notin H$. Let $Y$ be the subgroup generated in $\mathbf{Z} * \Gamma$ by $z g_{1}$. We have to prove that $Y$ and $H$ are free in $\mathbf{Z} * \Gamma$. Suppose that there exist $y_{i} \in Y-\{1\}$ and $h_{i} \in H-\{1\}$ such that $y_{1} h_{1} y_{2} h_{2} \ldots=1$. Choose such a product $P$ having a minimal number of $z$ 's and $z^{-1}$ 's in its decomposition. It's easy to see that $P$ is a product of $z, z^{-1}$ 's alternating with terms of the form $g_{1}, g_{1}^{-1}, h_{i}, g_{1} h_{i}, h_{i} g_{1}^{-1}$ or $g_{1} h_{i} g_{1}^{-1}$. As $P=1$ and $z$ is free from $\Gamma$, at least one of these terms has to be equal to 1 . But the only terms which can be equal to 1 are the ones of the form $g_{1} h_{i} g_{1}^{-1}$, which can only appear between a $z$ and a $z^{-1}$. Now by deleting $z g_{1} h_{i} g_{1}^{-1} z^{-1}$ from $P$ we get another product equal to 1 which has less $z$ 's and $z^{-1}$ 's, contradiction.

Remarks. For $A=C(G)$ with $G$ compact non-abelian the Woronowicz algebra $\tilde{A}$ is harder to describe. Note however that by $\mathbb{B} 2]$ we have $\tilde{C}(\mathbf{S U}(2))=A_{u}\left(I_{2}\right)$; in fact this result, as well as its generalisation $\tilde{A}_{o}(F)=$ $A_{u}(F)$ may be deduced from the Theorem 5.1.

For any pair $(A, u)$ satisfying (w1-3) let $A^{\prime}$ be the $\mathbf{C}^{*}$-subalgebra of $A$ generated by the the entries of the matrix $u^{\prime}:=\hat{u} \otimes u$. Then $\left(A^{\prime}, u^{\prime}\right)$ satisfies (w1-3) and it is easy to see that $\left(\tilde{A}^{\prime}, \tilde{u}^{\prime}\right)=\left(A^{\prime}, u^{\prime}\right)$. It is interesting to note that in the Proposition 5.3, $\tilde{\Gamma}$ is isomorphic to $\mathbf{Z} * H$ in all cases. This could be interpreted in the following way : if $(A, u)$ is of the form $\left(\mathbf{C}^{*}(\Gamma), \operatorname{diag}\left(u_{g_{1}}, \ldots, u_{g_{n}}\right)\right)$ then $\tilde{A} \simeq \mathbf{C}^{*}(\mathbf{Z}) * A^{\prime}$. This kind of free product decomposition of $\tilde{A}$ isn't aviable in general, but is valid for instance if the trivial representation is contained in an alternating product of the form $u \otimes \hat{u} \otimes \ldots \otimes \hat{u}$ (the proof is similar to the one of Prop. 5.3 (i)).

It is also possible in some of the remaining cases to obtain free product decompositions $v N(\tilde{A}) \simeq v N(\mathbf{Z}) * v N\left(A^{\prime}\right)$ at the level of von Neumann algebras (see the Theorem 6 in [B2]; the argument in its proof works for any $(A, u)$ such that the Haar measure of $A$ is a trace and such that the polar part of some coefficient of $u$ is unitary).

The simplicity results in [B2] may also be extended - if the trivial representation is not contained in any alternating product of the form $u \otimes \hat{u} \otimes \ldots \otimes \hat{u}$, 
one can show (first by studying the operation $\operatorname{Irr}(A) \mapsto \operatorname{Irr}(\tilde{A})$, then by applying the Prop. 8 in B2 $)$ that the reduced version of $\tilde{A}$ is simple, with at most one trace.

\section{Amenability}

In this section we prove a Kesten type result for the (discrete quantum groups represented by) Woronowicz algebras. This will give a characterisation of the amenable Popa systems of the form $\operatorname{End}\left(u^{\otimes[i, j]}\right)_{0 \leq i \leq j<\infty}$.

Definition 6.1 Let $A$ be a Woronowicz algebra and let $h \in A^{*}$ be its Haar measure. Define $A_{\text {red }}=A /\left\{x \in A \mid h\left(x^{*} x\right)=0\right\}$ and $A_{p}=$ envelopping $\mathrm{C}^{*}$-algebra of $A_{s}$. Then $A_{p}$ and $A_{\text {red }}$ are Woronowicz algebras, called the full and the reduced version of $A$ [W1], BSS. A is said to be amenable (as a Woronowicz algebra) if the canonical surjection $A_{p} \rightarrow A_{\text {red }}$ is an isomorphism.

Examples. If $\Gamma$ is a discrete group then the Woronowicz algebra $\mathbf{C}^{*}(\Gamma)$ is amenable if and only if $\Gamma$ is amenable. If $G$ is a compact group then the Woronowicz algebra $C(G)$ is clearly amenable (while this is not related to the fact that $G$ is amenable). See [BS], B] for the analogue of the Pontryagin duality and for the two dual notions of amenability.

We restrict the attention to the Woronowicz algebras satisfying (w1-3) for some corepresentation $u$ (these are the ones which represent the discrete quantum groups "of finite type").

Theorem 6.1 (G. Skandalis) Assume that $(A, u)$ satisfies (w1-3) with $u \in$ $M_{n}(A)$, and let $X \subset \mathbf{R}$ be the spectrum of $\operatorname{Re}(\chi(u)) \in A_{\text {red }}$.

(i) $X \subset[-n, n]$.

(ii) The Woronowicz algebra $A$ is amenable if and only if $n \in X$.

Proof. ( $i$ ) is clear, as all entries of the unitary $u$ are of norm $\leq 1$.

$(i i, \Longrightarrow)$ The counit of $A_{s}$ extends to the full version as a $\mathbf{C}^{*}$-morphism $A_{p} \rightarrow \mathbf{C}$ which sends $n-\operatorname{Re}(\chi(u)) \mapsto 0$. Thus $n-\operatorname{Re}(\chi(u))$ is not invertible in $A_{p}$, nor in $A_{\text {red }}=A_{p}$ if $A$ is amenable.

$(i i, \Longleftarrow)$ Embed isometrically $A_{\text {red }} \hookrightarrow B\left(l^{2}(A)\right)$ by the left regular representation. The matrix $u$ being unitary, its coefficients have norms $\leq 1$. In particular for all $i$ the operator $\alpha_{i}:=1-\operatorname{Re}\left(u_{i i}\right)$ is positive. 
Assume that $n$ is in the spectrum of $\operatorname{Re}(\chi(u))$, i.e. that the positive operator $\sum_{i} \alpha_{i}$ is not invertible. Then there exists a sequence $\xi_{k}$ of norm one vectors in $l^{2}(A)$ such that $<\left(\sum_{i} \alpha_{i}\right) \xi_{k}, \xi_{k}>\rightarrow 0$. As all $<\alpha_{i} \xi_{k}, \xi_{k}>$ 's are positive, we get $<\alpha_{i} \xi_{k}, \xi_{k}>\rightarrow 0$ for all $i$ and by using the equality

$$
\|(1-x) \xi\|^{2}=2<(1-\operatorname{Re}(x)) \xi, \xi>+\|x \xi\|^{2}-1
$$

valid for every $x$ and norm one vector $\xi$ we get $\left\|u_{i i} \xi_{k}-\xi_{k}\right\| \rightarrow 0$ for all $i$.

Now let $M_{n}\left(A_{\text {red }}\right)$ act on $\mathbf{C}^{n} \otimes l^{2}(A)$. As $u$ is unitary $\left\|u\left(e_{j} \otimes \xi_{k}\right)\right\|=1$ for all $j$ (where $\left\{e_{1}, \ldots, e_{n}\right\}$ is the canonical basis of $\mathbf{C}^{n}$ ). Thus $\sum_{i}\left\|u_{i j} \xi_{k}\right\|^{2}=1$, and as $\left\|u_{i i} \xi_{k}-\xi_{k}\right\| \rightarrow 0$ we get $\left\|u_{i j} \xi_{k}\right\| \rightarrow 0$ if $i \neq j$.

These results could be summarized as $\left\|u_{i j} \xi_{k}-\delta_{i j} \xi_{k}\right\| \rightarrow 0$ for all $i, j$. Now let $\varepsilon: A_{p} \rightarrow \mathbf{C}$ be the counit, $\pi: A_{p} \rightarrow A_{\text {red }}$ be the canonical projection and consider the set $M$ of elements of $A_{p}$ satisfying

$$
\left\|\pi(x) \xi_{k}-\varepsilon(x) \xi_{k}\right\| \rightarrow 0
$$

$M$ is clearly a closed $*$-subalgebra of $A_{p}$, and contains all the coefficients of $u$, so $M=A_{p}$ and the Proposition 5.5 in [B] shows that $A$ is amenable.

Theorem 6.2 If $v$ is a corepresentation of a Woronowicz algebra $A$ and if $B$ is the $\mathbf{C}^{*}$-subalgebra of $A$ generated by the coefficients of $v \otimes \hat{v}$ then the following are equivalent :

(i) $\operatorname{End}\left(v^{\otimes[i, j]}\right)_{0 \leq i \leq j<\infty}$ is amenable.

(ii) The Woronowicz algebra $B$ is amenable and its Haar measure is a trace.

Proof. Recall that a Popa $\lambda$-system is amenable if the norm of its principal graph is $\lambda^{-1 / 2}$. Let $\Gamma$ be the principal graph of $\operatorname{End}\left(v^{\otimes[i, j]}\right)_{0 \leq i \leq j<\infty}$ and $\lambda:=d(v)^{-2}$. Let also $u:=v \otimes \hat{v}$ and consider the lattice

$$
\operatorname{End}\left(u^{\otimes[i, j]}\right)_{0 \leq i \leq j<\infty}=\operatorname{End}\left(v^{\otimes[i, j]}\right)_{0 \leq i \leq j<\infty, i+j \text { even }}
$$

and denote by $\Gamma^{\prime}$ its principal graph. Then $\left\|\Gamma^{\prime}\right\|=\|\Gamma\|^{2}$, and $d(u)=d(v)^{2}$ by the Lemma 1.3, so the amenability of $\operatorname{End}\left(v^{\otimes[i, j]}\right)_{0 \leq i \leq j<\infty}$ is equivalent to the equality

$$
\left\|\Gamma^{\prime}\right\|=d(u)
$$

As $1 \in u=\hat{u}$ (as equivalence classes of corepresentations) we see that every element of $\operatorname{Irr}(B)$ is a subcorepresentation of $u^{\otimes n}, \forall n \geq N$, for some $N \in \mathbf{N}$. 
By identifying the vertices of $\Gamma^{\prime}$ with minimal central projections in the algebras $\operatorname{End}\left(u^{\otimes n}\right)$, thus with irreducible subcorepresentations of $u^{\otimes n}$, we see that both sets of even and odd vertices are equal to $\operatorname{Irr}(B)$. Thus the norm of $\Gamma^{\prime}$ is equal to the norm of the matrix

$$
M:=(\operatorname{dim}(\operatorname{Hom}(r \otimes u, p)))_{r, p \in \operatorname{Irr}(B)}
$$

Now $\operatorname{dim}(\operatorname{Hom}(r \otimes u, p))=<\chi(u) \chi(r), \chi(p)>$, where $<,>$ is the scalar product associated to the Haar measure on the algebra of characters $B_{\text {central }}$ (see W1]). Thus $M$ is the multiplication by $\chi(u)$ is $B_{\text {central }}$, so the norm of $M$ is equal to the norm of $\chi(u)$ in $B_{\text {red }}$. After all these identifications, we have the following equalities and inequalities (where the inequalities are clear) :

$$
\left\|\Gamma^{\prime}\right\|=\|M\|=\|\chi(u)\|_{B_{\text {red }}} \leq \operatorname{dim}(u) \leq d(u)
$$

Now $\operatorname{dim}(u)=d(u)$ iff the Haar measure of $B$ is a trace (see the example 1.1), and by the Th. $6.1\|\chi(u)\|_{B_{r e d}}=\operatorname{dim}(u)$ iff $B$ is amenable (note that $\chi(u)=\chi(v) \chi(v)^{*}$ is positive).

Corollary 6.1 If End $\left(v^{\otimes[i, j]}\right)_{0 \leq i \leq j<\infty}$ is amenable then its index is the square of an integer.

It is somehow clear from the proof of the Th. 6.2 that the notion of amenability of a Woronowicz algebra $A$ depends only on its corepresentation theory. This remark will give us the co-amenability of the $q$-deformations. More precisely, this will follow from following consequence of the Th. 6.1, which seems to have its own interest: it gives for instance information (a lower bound for dimensions) on the fiber functors on the monoidal category of corepresentations of an amenable Woronowicz algebra (see also the examples 1.3., 1.4, and the concluding remarks in the Introduction).

Proposition 6.1 Assume that $(A, u)$ and $(B, v)$ satisfy (w1-3) and that there exists an isomorphism of semirings $f: R^{+}(A) \rightarrow R^{+}(B)$ sending $u \mapsto v$. If the Woronowicz algebra $A$ is amenable then $\operatorname{dim}(f(r)) \geq \operatorname{dim}(r)$, $\forall r \in R^{+}(A)$, and the following conditions are equivalent.

(i) $\operatorname{dim}(f(r))=\operatorname{dim}(r), \forall r \in R^{+}(A)$.

(ii) $\operatorname{dim}(v)=\operatorname{dim}(u)$.

(iii) The Woronowicz algebra $B$ is amenable. 
Proof. Step I. We prove that $\operatorname{Spec}(\operatorname{Re}(\chi(u)))=\operatorname{Spec}(\operatorname{Re}(\chi(v)))$. By [W1 $R^{+}(A)$ and $R^{+}(B)$ are (as additive monoids) the free monoids on $\operatorname{Irr}(A)$ and $\operatorname{Irr}(B)$ respectively, and it follows that $f$ maps $\operatorname{Irr}(A)$ onto $\operatorname{Irr}(B)$. Let us prove now that $f$ is an isomorphism of involutive semirings, i.e. that $f(\hat{r})=\widehat{f(r)}$ for any $r \in R^{+}(A)$. For $r \in \operatorname{Irr}(A)$ this is clear, as $\hat{r}$ may be characterised as being the unique $p \in \operatorname{Irr}(A)$ such that there exists $q \in$ $R^{+}(A)$ with $r \otimes p=q+1$. The general case follows by complete reducibility.

Now by functional calculus the spectrum of $\operatorname{Re}(\chi(u)) \in A_{\text {red }}$ is the support of its spectral measure $\mu$ with respect to the Haar measure $h$ of $A_{\text {red }}$, which in turn is uniquely determined by its moments

$$
\mu\left(X^{k}\right)=h\left[\operatorname{Re}(\chi(u))^{k}\right]=2^{-k} \operatorname{dim}\left(\operatorname{Hom}\left(1,(u+\hat{u})^{\otimes k}\right)\right)
$$

hence by the isomorphism class of the pointed involutive semiring $\left(R^{+}(A), u\right)$. The equality of spectra follows.

Step II. We prove that $\operatorname{dim}(f(r)) \geq \operatorname{dim}(r), \forall r \in R^{+}(A)$. Firstly, by the Th. 6.1 and by the step $I$. we get $\operatorname{dim}(v) \geq \operatorname{dim}(u)$.

For any corepresentation $r$ of $A$ let $\mathbf{C}^{*}(r)$ be the $\mathbf{C}^{*}$-subalgebra of $A$ generated by the coefficients of $r$. Then (by fixing a basis making $r$ unitary) $\left(\mathbf{C}^{*}(r), r\right)$ satisfies (w1-3). As $A$ is amenable, $\mathbf{C}^{*}(r)$ is also amenable (see [BS], [B]], in fact this could be deduced also from the Theorem 6.1). The inequality $\operatorname{dim}(f(r)) \geq \operatorname{dim}(r)$ follows by replacing $(A, u)$ with $\left(\mathbf{C}^{*}(r), r\right)$.

Step III. The equivalences are clear: (i) $\Longrightarrow$ (ii) is trivial, (ii) $\Longrightarrow$ (iii) follows from the Th. 6.1 and from the step $I$., and if (iii) is satisfied, then by interchanging $(A, u)$ and $(B, v)$ we get the reverse inequalities in $(i)$.

Corollary 6.2 If $G$ is one of the groups $S U(n), S p(2 n)$ or $\operatorname{Spin}(n)$ and if $q>0$ then the Woronowicz algebra $C\left(G_{q}\right)$ is amenable.

Proof. By [R1], [R2] we have an isomorphism $R^{+}\left(C\left(G_{q}\right)\right) \simeq R^{+}(C(G))$ which preserves the dimensions. The assertion follows from (iii) $\Longleftrightarrow$ (i) in the Prop. 6.1 and from the fact that the Woronowicz algebra $C(G)$ is amenable (see the definition 6.1).

Note. For $S U(n)$ this was done by Nagy in $\mathbb{N}$. 


\section{References}

[BS] Baaj, S., Skandalis, G.: Unitaires multiplicatifs et dualité pour les produits croisés de $C^{*}$-algèbres. Ann. Sci. Ec. Norm. Sup. 26, 425-488 (1993)

[B1] Banica, T.: Théorie des représentations du groupe quantique compact libre $O(n)$. C. R. Acad. Sci. Paris 322, 241-244 (1996)

[B2] Banica, T.: Le groupe quantique libre $U(n)$. Commun. Math. Phys. (in press)

[Bi] Bisch, D.: Bimodules, higher relative commutants and the fusion algebra associated to a subfactor. Fields Inst. Commun. 13, 16-63 (1997)

[Bl] Blanchard, E.: Déformations de $C^{*}$-algèbres de Hopf. Bull. Soc. Math. Fr. 124, 141-215 (1996)

[D] Deligne, P.: Catégories tannakiennes. Grothendieck Festchrift, Vol. II, 111-195, Birkhauser (1990)

[DR] Doplicher, S., Roberts, J.: A new duality theory for compact groups. Invent. Math. 98, 157-218 (1989)

[EN] Enock, M., Nest, R.: Inclusions of factors, multiplicative unitaries and Kac algebras. J. Funct. Anal. 137, 466-543 (1996)

[G] Gurevich, D.I.: Algebraic aspects of the quantum Yang-Baxter equation. Leningrad Math. J. 2, 801-828 (1991)

[J1] Jones, V.: Fusion en algèbres de von Neumann et groupes de lacets (d'après A. Wassermann). Séminaire Bourbaki $n^{\circ} 800$ (1995)

[J2] Jones, V.: to appear.

[KW] Kazhdan, D., Wenzl, H.: Reconstructing monoidal categories. I.M. Gelfand Seminar, Adv. in Soviet Math. vol. 16, part 2, 111-136 (1993)

[N] Nagy, G.: On the Haar measure of the quantum $S U(N)$ group. Commun. Math. Phys. 153, 217-228 (1993)

[NS] Nica, A., Speicher, R.: R-diagonal pairs - a common approach to Haar unitaries and circular elements. Fields Inst. Commun. 12, 149-188 (1997) 
[P1] Popa, S.: Sousfacteurs, actions des groupes et cohomologie. C. R. Acad. Sci. Paris 309, 771-776 (1992)

[P2] Popa, S.: Markov traces on universal Jones algebras and subfactors of finite index. Invent. Math. 111, 375-405 (1993)

[P3] Popa, S.: Classification of amenable subfactors of type II. Acta Math. $172,163-255$ (1994)

[P4] Popa, S.: An axiomatization of the lattice of higher relative commutants of a subfactor. Invent. Math. 120, 427-445 (1995)

[PW] Popa, S., Wassermann, A.: Actions of compact Lie groups on von Neumann algebras. C. R. Acad. Sci. Paris 315, 421-426 (1992)

[R1] Rosso, M.: Finite dimensional representations of the quantum analog of the enveloping algebra of a complex semisimple Lie algebra. Commun. Math. Phys. 117, 581-593 (1988)

[R2] Rosso, M.: Algèbres enveloppantes quantifiées, groupes quantiques compacts de matrices et calcul differentiel non-commutatif. Duke Math. J. 61, 11-40 (1990)

[TL] Toledano Laredo, V.: Fusion of positive energy representations of $\operatorname{LSpin}_{2 n}$. PhD thesis, Univ. of Cambridge (1997)

[VDW] Van Daele, A., Wang, S.Z.: Universal quantum groups. International J. of Math. Vol. 7, No. 2, 255-264 (1996)

[VDN] Voiculescu, D., Dykema, K., Nica, A.: Free random variables. CRM Monograph Series $n^{\circ} 1$, AMS (1993)

[Wn] Wang, S.Z.: Free products of compact quantum groups. Commun. Math. Phys. 167, 671-692 (1995)

[Ws] Wassermann, A.: Coactions and Yang-Baxter equations for ergodic actions on subfactors. LMS Lecture Notes 136, 203-236 (1988)

[We1] Wenzl, H.: Hecke algebras of type $A_{n}$ and subfactors. Invent. Math. 92, 349-383 (1988) 
[We2] Wenzl, H.: Quantum groups and subfactors of type B,C, and D. Commun. Math. Phys. 133, 383-432 (1990)

[We3] Wenzl, H.: $C^{*}$ tensor categories from quantum groups. preprint (1996)

[W1] Woronowicz, S.L.: Compact matrix pseudogroups. Commun. Math. Phys. 111, 613-665 (1987)

[W2] Woronowicz, S.L.: Tannaka-Krein duality for compact matrix pseudogroups. Twisted $S U(n)$ groups. Invent. Math. 93, 35-76 (1988)

[W3] Woronowicz, S.L.: Compact quantum groups. preprint

[X] Xu, F.: Standard $\lambda$-lattices from quantum groups. preprint (1996) 